University of Windsor

Scholarship at UWindsor

$12-3-2010$

\title{
Nicholas Reactions in the Construction of Cyclohepta[de]naphthalenes and Cyclohepta[de]naphthalenones. The Total Synthesis of Microstegiol
}

\author{
RAFIQ TAJ \\ University of Windsor \\ James R. Green \\ University of Windsor
}

Follow this and additional works at: https://scholar.uwindsor.ca/chemistrybiochemistrypub

Part of the Biochemistry, Biophysics, and Structural Biology Commons, and the Chemistry Commons

\section{Recommended Citation}

TAJ, RAFIQ and Green, James R.. (2010). Nicholas Reactions in the Construction of Cyclohepta[de]naphthalenes and Cyclohepta[de]naphthalenones. The Total Synthesis of Microstegiol. Journal of Organic Chemistry, 75 (23), 8258-8270.

https://scholar.uwindsor.ca/chemistrybiochemistrypub/75

This Article is brought to you for free and open access by the Department of Chemistry and Biochemistry at Scholarship at UWindsor. It has been accepted for inclusion in Chemistry and Biochemistry Publications by an authorized administrator of Scholarship at UWindsor. For more information, please contact scholarship@uwindsor.ca. 


\title{
Nicholas reactions in the construction of
}

\author{
cyclohepta[de]naphthalenes and
}

\section{cyclohepta[de]naphthalenones. Preparation of the microstegiol ring system}

Rafiq A. Taj and James R. Green*

Department of Chemistry and Biochemistry, University of Windsor, Windsor, ON, N9B 3P4, Canada

jgreen@uwindsor.ca

RECEIVED DATE (to be automatically inserted after your manuscript is accepted if required according to the journal that you are submitting your paper to)

Tel: (519)-253-3000, Ext. 3545. Fax: (519)-973-7098

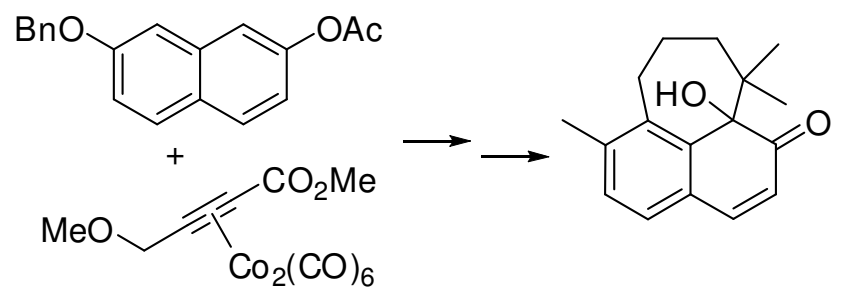

The application of the Nicholas reaction chemistry of 2,7-dioxygenated naphthalenes towards the synthesis of cyclohepta[de]napthalenes, particularly the rearranged abietane ring system of microstegiol, is presented. The substitution profile of Nicholas monosubstitution (predominantly C-1) and disubstitution reactions (predominantly 1,6-) on 2,7-dioxygenated napthalenes is reported. Application of a 1,8-dicondensation product and selected $\mathrm{C}-1$ monocondensation products to the construction of 
cyclohepta[de]naphthalenes by way of ring closing metathesis and intramolecular Friedel-Crafts reactions, respectively, are described. Deprotection of the C-7 oxygen function to the corresponding naphthol allows tautomerization to cyclohepta[de]naphthalene-1-ones upon seven membered ring closure in most cases, and replacement of the C-2 oxygen function in the naphthalene by a methyl group ultimately allows the synthesis of model compound 38, lacking only the isopropyl group of microstegiol.

\section{Introduction}

The cyclohepta[de]naphthalenes comprise a class of seven membered ring compounds that has received significant attention for decades. Much of the early work centered around the pleiadienes and acepleiadylenes and stemmed from interest in their electronic structure. More recently, several rearranged abietanes containing the cyclophepta[de]naphthalene nucleus, including microstegiol (1), ${ }^{1}$ oxomicrostegiol (2), ${ }^{2}$ salvibretol (3), ${ }^{1 \mathrm{c}}$ and oxosalvibretol (4), ${ }^{1 \mathrm{c}, \mathrm{m}}$ have been isolated from roots of a number of plants of the genus Salvia. These plants have often seen use as folk remedies, and their crude extracts have demonstrated antibacterial, anticancer, and insect antifeedant activities. ${ }^{1 \mathrm{~h}, \mathrm{k}, 3}$ Microstegiol itself has been demonstrated to have antileukemic activity and modest antibacterial activity. ${ }^{1 a, 1 k, 1 \mathrm{~m}, \mathrm{ln}}$

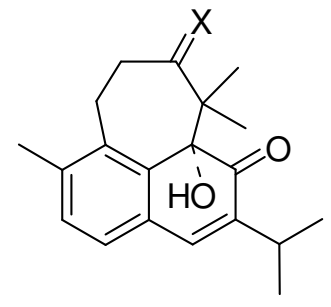

1 microstegiol, $\mathrm{X}=\mathrm{H}_{2}$

2 oxomicrostegiol, $\mathrm{X}=\mathrm{O}$

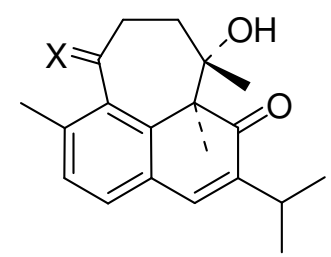

3 salvibretol, $\mathrm{X}=\mathrm{H}_{2}$

4 oxosalvibretol, $\mathrm{X}=\mathrm{O}$

Despite the recent upsurge in interest in the natural product literature, none of the rearranged abietanes 1-4 have been the subject of synthetic activity. The carbon framework of the cyclohepta[de]naphthalenes themselves have normally been prepared by sequential Friedel-Crafts acylations of succinic anhydride or some variation of that approach, ${ }^{4}$ although scattered reports of access to this ring system by $2+2$ cycloaddition/ring expansion reactions of cyclopenta[de]naphthalenes, ${ }^{5}$ 
malonate intramolecular alkylation processes, ${ }^{6}$ reductive carbonyl coupling reactions, ${ }^{7}$ and $o$-xylylene Diels-Alder cycloadditions ${ }^{8}$ have been published. ${ }^{9}$

Our group has a long-standing interest in the synthesis of seven-membered ring compounds, ${ }^{10}$ based in part on the ability of alkynedicobalt complexes and the Nicholas reaction chemistry ${ }^{11}$ of their derived propargyldicobalt cations to allow umpolung bond constructions from reactions of -carbonyl cation equivalents with electron rich arenes or other nucleophiles..$^{12,13,14,15}$ Given the recent interest in 1-4 and the relative paucity of methods for the synthesis of cyclohepta[de]naphthalenes, we considered it of importance to study the applicability of these cations to this ring system, with a particular attention to the tautomerized naphthol (cyclohepta[de]naphthalene-1-one) framework of microstegiol (1). Given its structural characteristics, we consider the issues of a) the fundamental substitution characteristics in Nicholas reactions on 2,7-disubstituted naphthalenes, b) seven membered ring construction derived from Nicholas reaction adducts, c) tautomerization to the keto- form of the 7- (or 2-) hydroxynaphthalenes, and d) regioselection in the constructed cycloheptane to be significant for the methodology. This work describes in complete form our developments in these directions. ${ }^{16}$

\section{Results and Discussion}

We believed it necessary to develop a comprehensive understanding of the pattern of reactivity of propargyldicobalt cations with naphthalene-2,7-diol derivatives. To this end we chose 2,7dimethoxynaphthalene (5a) as a reference molecule and studied the Lewis acid mediated Nicholas reactions of a number of substituted propargyl alcohol- or propargyl ether $\mathrm{Co}_{2}(\mathrm{CO})_{6}$ complexes $(\mathbf{6 a - f})$.

Monocondensation. The condensation reactions of 6 with 2,7-dimethoxynaphthalene (5a) occurred to variable levels of conversion in the presence of stoichiometric amounts of $\mathrm{BF}_{3}-\mathrm{OEt}_{2}$, but readily to complete conversion in the presence of 3 equiv of $\mathrm{BF}_{3}-\mathrm{OEt}_{2}$ (Table 1). With approximately equimolar amounts of $\mathbf{6 a - f}$ (1.1 equiv) relative to $\mathbf{5 a}$, monocondensation products $(\mathbf{7 , 8})$ were formed in most cases without significant contamination from the products of dialkylation. Nicholas reaction precursors (6a-d) bearing no substitution at the propargylic site cleanly afforded C-1 substitution in good yields, regardless of whether the remote site of the alkyne bore no substitution (7a, 93\%), or substitution by an 
electron withdrawing group (7b, 76\%), an alkyl group (7c, 84\%) or a silyl group (7d, 97\%). Substitution at the propargylic site by a methyl group (6e) still allowed C-1 substitution to occur (7e, 66\%), but phenyl substitution at the propargyl site (6f) resulted in condensation to give C-3 substitution product (8f, 51\%) as the sole isolable one. Use of 2,7-dibenzyloxysubstituted $\mathbf{5 b}$ gave an analogous reaction with ester substituted complex $\mathbf{6 b}$, with the exception of giving a small amount of 1,8-dicondensation product $9 \mathrm{~g}(13 \%)$ in addition to the $\mathrm{C}-1$ monocondensation product $\mathbf{7 g}(71 \%)$.

Table 1. Monocondensation reactions of $6 \mathbf{a - f}$ with $\mathbf{5 a - b}$.

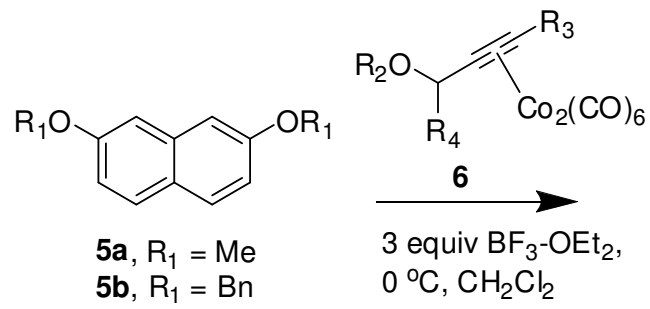

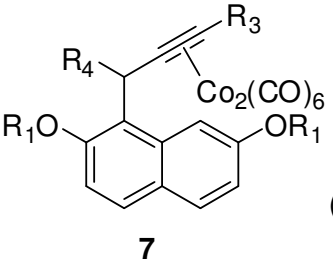

7

6-8 $\left(R_{2}=H\right)$, except $6 b, R_{2}=M e$ 7-8a-f $\left(R_{1}=H\right), 7 g, R_{1}=B n$ d, $\mathrm{R}_{3}=\mathrm{SiMe}_{3}, \mathrm{R}_{4}=\mathrm{H}$

e, $R_{3}=H, R_{4}=M e$

f, $\mathrm{R}_{3}=\mathrm{H}, \mathrm{R}_{4}=\mathrm{Ph}$

g, $\mathrm{R}_{3}=\mathrm{CO}_{2} \mathrm{Me}, \mathrm{R}_{4}=\mathrm{H}$

\begin{tabular}{|l|l|l|l|l|}
\hline Entry & $\mathbf{5}$ & $\mathbf{6}$ & $\mathbf{7}$ (yield, \%) & $\mathbf{8}$ (yield, \%) \\
\hline 1 & $\mathbf{5 a}$ & $\mathbf{6 a}$ & $\mathbf{7 a}(93)$ & \\
\hline 2 & $\mathbf{5 a}$ & $\mathbf{6 b}$ & $\mathbf{7 b}(88)$ & \\
\hline 3 & $\mathbf{5 a}$ & $\mathbf{6 c}$ & $\mathbf{7 c}(84)$ & \\
\hline 4 & $\mathbf{5 a}$ & $\mathbf{6 d}$ & $\mathbf{7 d}(97)$ & \\
\hline 5 & $\mathbf{5 a}$ & $\mathbf{6 e}$ & $\mathbf{7 e}(66)$ & \\
\hline 6 & $\mathbf{5 a}$ & $\mathbf{6 f}$ & - & $\mathbf{8 f}(51)$ \\
\hline 7 & $\mathbf{5 b}$ & $\mathbf{6 b}$ & $\mathbf{7 g}(71)^{\mathrm{a}}$ & \\
\hline
\end{tabular}

\footnotetext{
${ }^{\mathrm{a}}$ In addition, $13 \%$ of $\mathbf{9 g}$ was isolated.
} 
Dicondensation. Increasing the amount of propargyl alcohol/ether- $\mathrm{Co}_{2}(\mathrm{CO})_{6}$ complex 6a-e to slightly over two equivalents enabled, in most cases, ready conversion of 2,7-dimethoxynaphthalene (5a) to disubstituted products (9-10) (Table 2). In these cases, a competition existed between the formation of 1,8-disubstitution products (9) and 1,6-disubtitution products (10), which depended upon the structure of the Nicholas reaction precursor complex 6. In the case of unsubstituted $\mathbf{6 a}, 1,8$-disubstitution predominated, although not to the exclusion of 1,6-disubstitution (9a, 63\%, 10a, 9\%). This tendency toward 1,8-disubstitution was increased in the case of remote electron withdrawing group substituted $\mathbf{6 b}$, as $9 \mathbf{b}(86 \%)$ was formed to the exclusion of any 1,6-disubstitution product. Conversely, substitution at the remote end of the alkyne complex with either methyl (6c) or trimethylsilyl (6d) groups resulted in the second substitution occurring at C-6, giving 1,6-disubstitution products 10c (86\%) and 10d (40\%); in the case of $\mathbf{6 d}$, the second substitution was incomplete, despite allowing the reaction to warm to rt, and a significant amount of $\mathbf{7 d}(59 \%)$ was also isolated.

Table 2. Dicondensation reactions of $\mathbf{6}$ with $\mathbf{5 a}$.

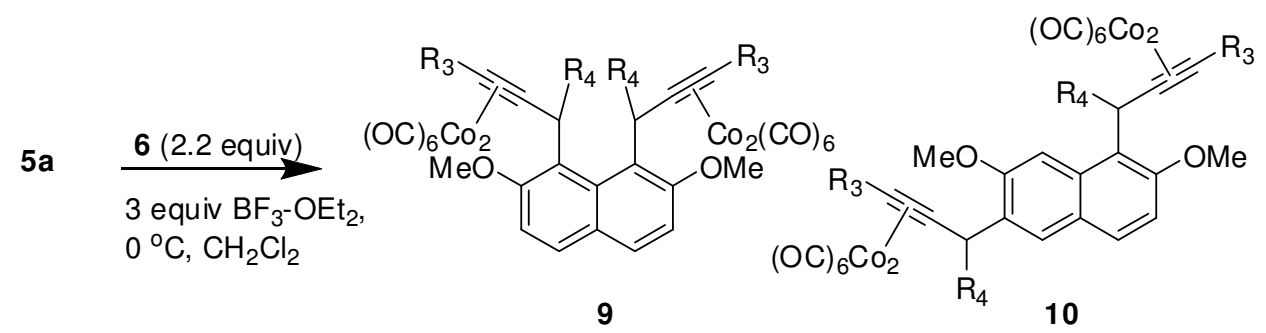
a, $\mathrm{R}_{3}=\mathrm{R}_{4}=\mathrm{H}$
b, $\mathrm{R}_{3}=\mathrm{CO}_{2} \mathrm{Me}, \mathrm{R}_{4}=\mathrm{H}$
c, $R_{3}=M e, R_{4}=H$
d, $\mathrm{R}_{3}=\mathrm{SiMe}_{3}, \mathrm{R}_{4}=\mathrm{H}$
e, $R_{3}=H, R_{4}=M e$
f, $\mathrm{R}_{3}=\mathrm{H}, \mathrm{R}_{4}=\mathrm{Ph}$
g, $\mathrm{R}_{3}=\mathrm{CO}_{2} \mathrm{Me}, \mathrm{R}_{4}=\mathrm{H}$

\begin{tabular}{|l|l|l|l|l|}
\hline Entry & $\mathbf{5 a}$ & $\mathbf{6}$ & $\mathbf{9}($ yield, \%) & $\mathbf{1 0}($ yield, \%) \\
\hline 1 & $\mathbf{5 a}$ & $\mathbf{6 a}$ & $\mathbf{9 a}(63)$ & $\mathbf{1 0 a}(9)$ \\
\hline 2 & $\mathbf{5 a}$ & $\mathbf{6 b}$ & $\mathbf{9 b}(86)$ & - \\
\hline 3 & $\mathbf{5 a}$ & $\mathbf{6 c}$ & - & $\mathbf{1 0 c}(86)$ \\
\hline 4 & $\mathbf{5 a}$ & $\mathbf{6 d}$ & - & $\mathbf{1 0 d}(40)^{\mathrm{a}}$ \\
\hline
\end{tabular}

\footnotetext{
${ }^{\mathrm{a}}$ In addition, $59 \%$ of $\mathbf{7 d}$ was isolated.
} 
In addition, two of the monocondensation products were taken and subjected to a second Nicholas reaction (Scheme 1). C-1 substitution product 7a was subjected to $\mathrm{BF}_{3}-\mathrm{OEt}_{2}$ mediated reaction with butyn-2-ol complex 6e, giving 10e in 72\% yield. C-3 substituted 8f, under analogous conditions with unsubstituted 6a, afforded $10 \mathbf{f}$ (50\% yield).

SCHEME 1. Mixed dicondensation products.

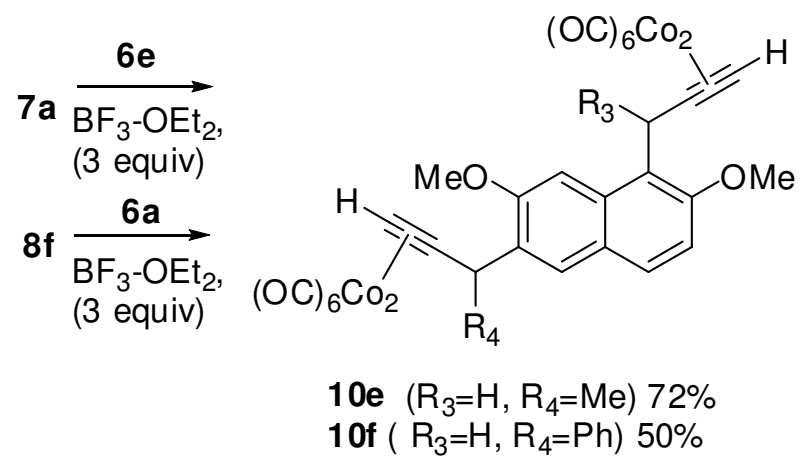

The pattern of Nicholas reaction reactivity of 2,7-dimethoxynaphthalene is therefore relatively consistent. Propargyldicobalt cations which are unsubstituted or substituted with electron withdrawing groups undergo $\mathrm{C}-1$ monosubstitution and $\mathrm{C}-1, \mathrm{C}-8$ disubstitution. If the cation possesses an alkynyl substitutent that is not electron withdrawing, monosubstitution is at $\mathrm{C}-1$, while the second substitution occurs at C-6. Substitution of $\mathbf{5}$ with groups at the propargylic site allow C-1 monosubstitution if the group is of moderate size, while a group of sufficient size at this site ultimately forces the initial Nicholas reaction to occur at C-3. The dichotomy in disubstitution is reminiscent of that of dinitration and chlorination (1,8-) versus dibromination $(1,6-)$ of 2,7 -dimethoxynaphthalene, ${ }^{17}$ including the element of the most electrophilic reagents being directed toward the 1,8- positions.

Application Towards Cyclohepta[de]naphthalene Synthesis. Given an understanding of the substitution patterns of 2,7-dioxygenated naphthalenes towards propargyldicobalt cations, three different approaches to construction of the cyclohepta[de]naphthalene ring system were envisaged. Use of dicondensation product 9a was the most readily apparent precursor, as it was one of the few cases in which the naphthalene 1,8-disubstitution pattern incorporated within the cyclohepta[de]naphthalene 
predominated; consequently its use in the preparation of the cyclohepta[de]naphthalene system was pursued (Scheme 2). Decomplexation of 9a could be accomplished with $\left(\mathrm{NH}_{4}\right)_{2} \mathrm{Ce}\left(\mathrm{NO}_{3}\right)_{6}$ under carefully controlled, low temperature conditions, giving $\mathbf{1 1}$ (57\% yield, 89\% by recovered starting material (brsm)). Other common reagents for decomplexation $\left(\mathrm{Me}_{3} \mathrm{NO}, \mathrm{I}_{2}\right)$ gave poorer yields of $\mathbf{1 1}$ or none at all. Semi-hydrogenation of $\mathbf{1 1}$ in the presence of the Lindlar catalyst then afforded diene $\mathbf{1 2}^{18}$ (89\% yield, 98\% brsm). Finally, ring closing metathesis (RCM) employing Grubbs 1 catalyst (5 mol\%) gave dihydrocyclohepta[de]naphthalene 13 (85\% yield). While this work was in progress, the Kotha and Chattopadhyay groups demonstrated the accessibility of the corresponding acetates by RCM. ${ }^{19}$

SCHEME 2. Cyclohepta[de]naphthalenes by RCM.

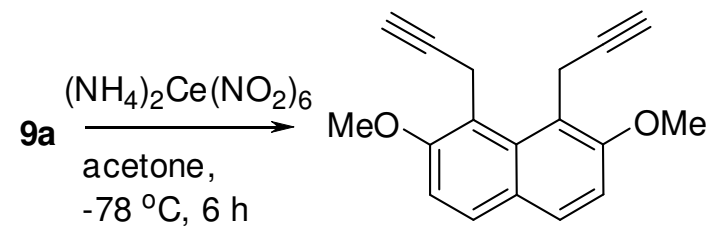

11, 57\% (89\% brsm)

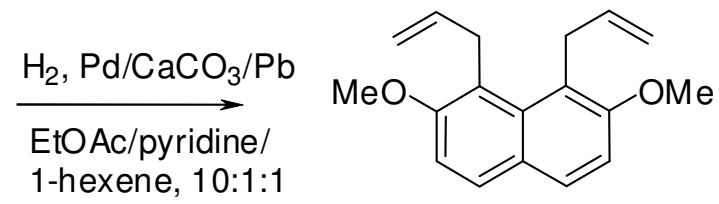

$12,89 \%$

(98\% brsm)

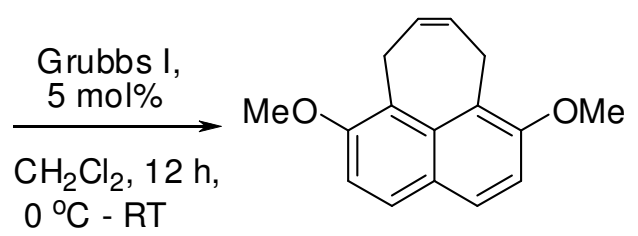

$13,85 \%$

Using this dipropargylation-RCM approach to cycloheptanaphthalenes, however, afforded only limited possibilities for creating unsymmetrical substitution patterns regioselectively on both the naphthalene and cycloheptene portions of $\mathbf{1 3}$, due to the incompatibility of propargyl substitution in $\mathbf{6}$ with 1,8-disubstitution on $\mathbf{5}$. Consequently, the applicability of monocondensation product $\mathbf{7 b}$ toward cyclohepta[de]naphthalene construction was explored (Scheme 3). Decomplexation of the organic unit from the $\mathrm{Co}_{2}(\mathrm{CO})_{6}$ residue of $\mathbf{7 b}$ was readily accomplished by treatment of iodine in THF, giving 14 in excellent yield (98\%). While hydrogenation of $\mathbf{1 4}$ over $\mathrm{Pd} / \mathrm{C}$ was sluggish, reduction of the triple bond 
to the alkanoate (15) was straightforward in the presence of $\mathrm{H}_{2}$ and $\mathrm{Rh} / \mathrm{C}$ (99\% yield). Addition of excess MeLi (7 equiv) to $\mathbf{1 5}$ resulted in attack on the ester function to give tertiary alcohol $\mathbf{1 6}$ in high yield (90\% yield) provided a significant excess of MeLi was employed; more modest excesses (i.e., 3 equiv) resulted in noticeable amounts of ketone 17 being isolated in addition to 16. Subjecting tertiary alcohol 16 to $\mathrm{H}_{2} \mathrm{SO}_{4}$ in $\mathrm{CH}_{2} \mathrm{Cl}_{2}$ induced its conversion to 7,7dimethyltetrahydrocyclohepta[de]naphthalene $\mathbf{1 8}$ (70\% yield), contaminated with a small amount of elimination product $\mathbf{1 9}$ (8\% yield).

SCHEME 3. Ring closure by Friedel-Crafts alkylation.
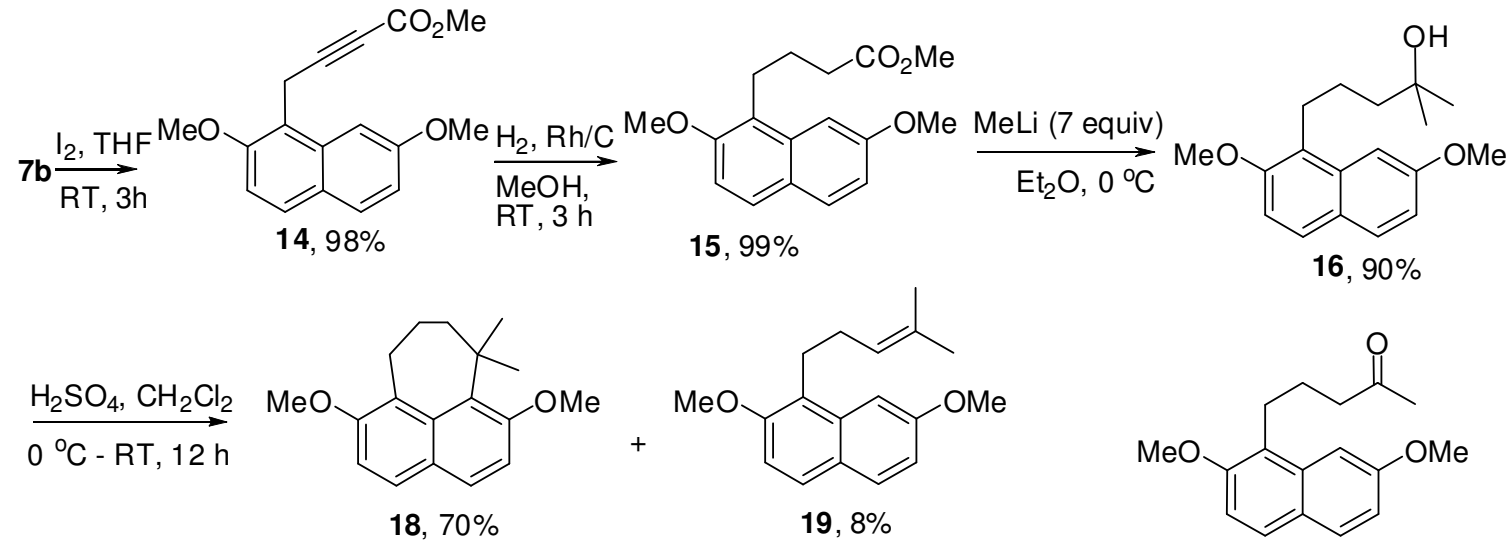

$16,90 \%$

As all the naturally occurring cyclohepta[de]napthalenes exist as dehydrotetralones, and since the matter of selective deprotection of the methoxy groups of $\mathbf{1 8}$ is non trivial, related approaches to these ring systems were made on phenolic acetate $20 .{ }^{20}$ The Nicholas reaction of $\mathbf{2 0}$ with $\mathbf{6 b}$ in the presence of $\mathrm{BF}_{3}-\mathrm{OEt}_{2}$ occurred somewhat sluggishly (relative to 2,7-dimethoxybenzene), but ultimately afforded the product of - carbonyl cation substitution ortho- to the methoxy function (21) in excellent yield (88\%) (Scheme 4). $\mathrm{Bu}_{2} \mathrm{BOTf}$ has often proved more effective in generating -carbonyl cation based Nicholas reactions, and accordingly, this Lewis acid enabled the same transformation to occur more rapidly, even in substoichiometric amounts, ultimately giving 21 in similar yields (90\%). ${ }^{21}$ Alkyne decomplexation with $\mathrm{I}_{2}$ in THF occurred readily to give alkynoate $22(93 \%)$, and $\mathrm{Pd} / \mathrm{C}$ catalyzed hydrogenation of the alkyne afforded alkanoate $\mathbf{2 3}$ in excellent yield (93\%). 
From alkanoate $\mathbf{2 3}$, the synthesis of cyclohepta[de]naphthalene systems diverged in two ways. Given the oxygenated cycloheptane unit present within salvibretol, access to a ketone function in the seven membered ring was desired. Consequently, saponification of $\mathbf{2 3}$ was accomplished, albeit slowly, by $\mathrm{K}_{2} \mathrm{CO}_{3}$ in $\mathrm{MeOH}$ at reflux, giving 24 (70\% yield). After experimentation with several sets of acidic conditions, it was found that exposure to polyphosphoric acid (PPA) $\left(\mathrm{CH}_{2} \mathrm{Cl}_{2}\right.$, reflux) gradually converted $\mathbf{2 4}$ to $\mathbf{2 5}$ (80\% yield). Compound $\mathbf{2 5}$ was found to exist entirely as the phenolic tautomer, the conjugation of the aromatic system to the ketone no doubt being in part responsible; the existence of 1hydroxy-7,12-pleiadenedione and related compounds in their naphthol tautomer provides additional precedent for this observation. $^{22}$

SCHEME 4. Ring closure by Friedel-Crafts acylation.


In contrast to 25, PM5 and DFT (B88-PW91 functional, dzvp basis set) calculations suggest that without the ketone function in the cycloheptane unit, the 1-hydroxycyclohepta[de]naphthalene cyclohepta[de]naphthalen-1(7H)-one equilibrium strongly favors the latter tautomer. As a result, 23 was subjected to reaction with excess MeLi, to give tertiary alcohol 26 (70\% yield) (Scheme 5). Subsequent addition of one drop of $\mathrm{H}_{2} \mathrm{SO}_{4}$ to a $\mathrm{CH}_{2} \mathrm{Cl}_{2}$ solution of this phenol at $0{ }^{\circ} \mathrm{C}$ induced rapid closure of the seven membered ring, producing 27 (70\% yield) exclusively as its keto tautomer. This angle straindirected tautmerization demonstrates the viability of the approach for preparing the rearranged abietane framework of microstegiol. 
SCHEME 5. Cyclohepta[de]napthalenone synthesis by cyclization-tautomerization.

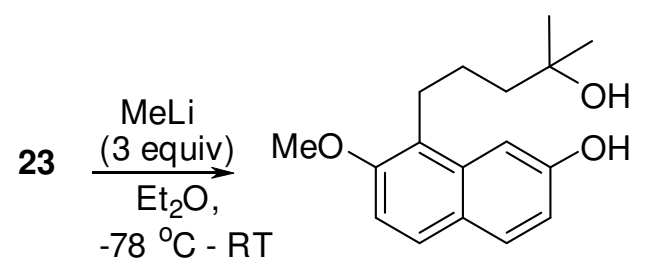

26, $70 \%$

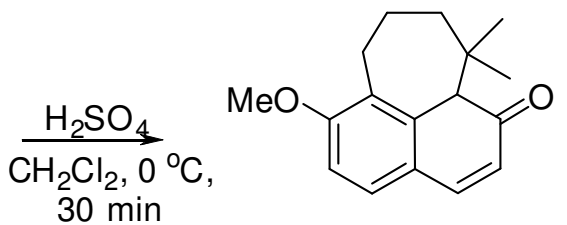

27, $70 \%$

Methyl Group Incorporation. In the naturally occurring cyclohepta[de]naphthalenes, there resides a C-6 methyl function (C-2 in the source naphthalene) as opposed to an oxygen based one. As a strongly electron donating function is required for viable Nicholas reaction/ -carbonyl cation chemistry on the naphthalene nucleus, its ultimate replacement by methyl is necessary. To that end, we chose benzyl/acetyl protected 28, prepared from 7-benzyloxy-2-naphthol, as a starting point for further studies (Scheme 6). Compound 28 underwent reaction with $\mathbf{6 b}$ only sluggishly in the presence of $\mathrm{BF}_{3}-\mathrm{OEt}_{2}$ (24h, $0{ }^{\circ} \mathrm{C}, 45 \%$ yield of $\mathbf{2 9}, 66 \%$ brsm), but gave 29 promptly and in excellent yield in the presence of $\mathrm{Bu}_{2}$ BOTf $\left(1.5 \mathrm{~h}, 0{ }^{\circ} \mathrm{C}, 90 \%\right.$ yield). Following uneventful decomplexation with $\mathrm{I}_{2}(\mathbf{3 0}, 90 \%$ yield), hydrogenation under $\mathrm{Rh} / \mathrm{C}$ catalysis reduced the triple bond without affecting the benzyl protecting group (31, 92\% yield); subsequent treatment of $\mathbf{3 1}$ with $\mathrm{H}_{2}$ and $\mathrm{Pd} / \mathrm{C}$ then gave phenolic acetate $\mathbf{3 2}$ in excellent yield (96\%). Attempts at using $\mathrm{H}_{2}$ with $\mathrm{Pd} / \mathrm{C}$ on $\mathbf{3 0}$ to effect a one pot conversion to 32 resulted in less clean reaction mixtures, which did contain some 32. Compound 32 was converted readily into its triflate $(33,96 \%)$ in the presence of $\mathrm{Tf}_{2} \mathrm{O} /$ pyridine, which was subjected to methylation. After some experimentation, it was found that $\operatorname{Pd}_{2}(\mathrm{dba})_{3}$ catalyzed reaction of $\mathbf{3 3}$ with the DABCO$\left(\mathrm{AlMe}_{3}\right)_{2}$ complex (DABAL-Me $)^{23}$ gave efficient methyl group incorporation, resulting in the formation of both $34(84 \%)$ and a small amount of deacetylated $35(7 \%)$.

SCHEME 6. C-2 methyl group incorporation. 


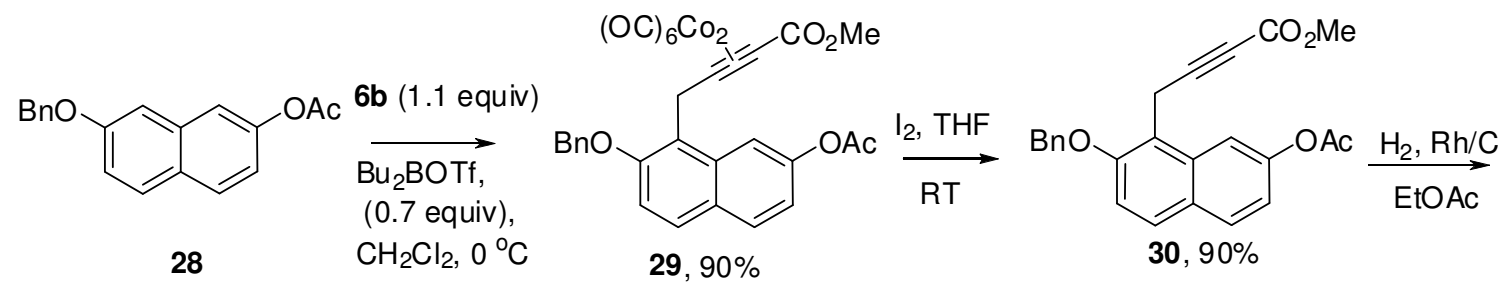<smiles>CC(=O)Oc1ccc2ccc(OC(C)=O)c(CCCC(=O)O)c2c1</smiles><smiles>CC(=O)CCCc1c(C)ccc2ccc(OC(C)=O)cc12</smiles>

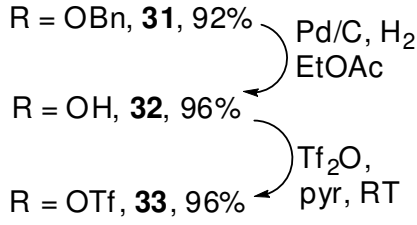

34, $84 \%$ $\mathrm{Pd}_{2}(\mathrm{dba})_{3}(1.5 \mathrm{~mol} \%)$, DABAL-Me 3 , THF, $\triangle$
$35,7 \%$

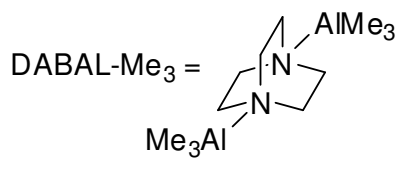

Each of these methylnaphthalenes could be converted into naphthol-tertiary alcohol $\mathbf{3 6}$ by reaction with excess MeLi (Scheme 7). In the case 34, 36 was obtained in 94\% yield; in the case of 35, 36 could be isolated in $70 \%$ yield. Subjecting 36 in $\mathrm{CH}_{2} \mathrm{Cl}_{2}$ to $\mathrm{H}_{2} \mathrm{SO}_{4}$ then resulted in rapid conversion to cyclohepta[de]naphthalenone $\mathbf{3 7}$ in excellent yield (87\%).

SCHEME 7. Preparation of cyclohepta[de]naphthalenone 37.

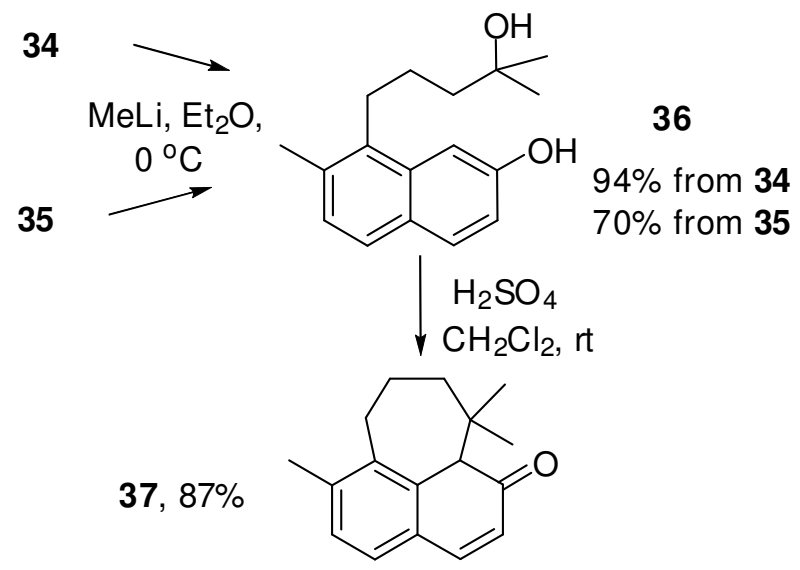

The incorporation of an -hydroxy function relative to the ketone was briefly explored (eq 1). Exposure of a solution of $\mathbf{3 7}$ in DMF to $\mathrm{NaH}$ in air $^{24}$ gave resulted in the disappearance of $\mathbf{3 7}$ and the formation of 2 compounds; -hydroxy ketone 38 (42\%), and the relatively unstable -hydroxylated epoxide $39(31 \%)$. 


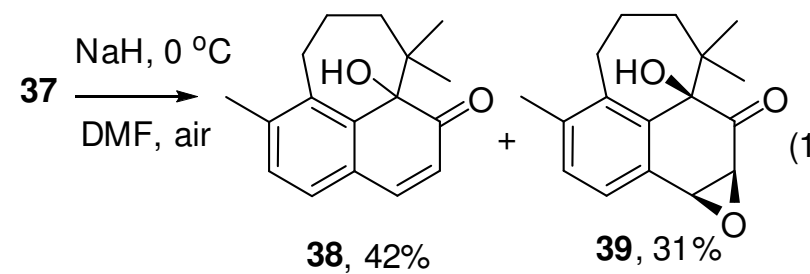

In summary, a systematic study of the applicability of Nicholas reaction based -carbonyl cation chemistry on 2,7-dioxygenated naphthalenes has demonstrated a preference for C-1 over C-3 monosubstitution in all but the most sterically hindered cases, and with a preference for $\mathrm{C}-1 / \mathrm{C}-6$ disubstitution, except in the least sterically hindered or most electron deficient cases. While the 1,8 dipropargylated case may be converted to cyclohepta[de]naphthalenes by way of RCM, the $\mathrm{C}-1$ carbonyl cation adducts may be converted to the cyclohepta[de]naphthalenes by a cyclization based on Friedel-Crafts acylation or alkylation; in the latter case this allows tautomerization of the naphthol to cyclohepta[de]naphthalen-1-one. Replacement of the C-2 oxygen function of the naphthalene by a methyl group after Nicholas reaction allows formation of the framework of microstegiol, including the -hydroxy function. Future work will be targeted towards an early introduction of an isopropyl function, and ultimately the synthesis of microstegiol itself.

Acknowledgements. The authors are grateful to NSERC (Canada), the Canada Foundation for Innovation (CFI), and the Ontario Innovation Trust (OIT) for support of this research.

Supporting Information Available. ${ }^{1} \mathrm{H}$ and ${ }^{13} \mathrm{C}$ NMR spectra for all new compounds. This material is available free of charge via the Internet at http://pubs.acs.org.

\section{Experimental Section.}

The General Experimental Methods follow a previous report from our laboratory. ${ }^{12 a}$ Starting materials $5 \mathbf{b},{ }^{25} 6 \mathbf{a},{ }^{26} 6 \mathbf{b},{ }^{27} 6 \mathbf{c},{ }^{28} 6 \mathbf{d},{ }^{29} 6 \mathbf{e},{ }^{30} 6 \mathbf{f}^{29}$ and $20^{31}$ were prepared by a literature procedures.

Hexacarbonyl[ $\mu$ - 4-(2,7-dimethoxy-1-(prop-2-ynyl)naphthalene)]dicobalt (7a). Method 1: To a solution of 2,7-dimethoxynaphthalene $5 \mathbf{a}(0.050 \mathrm{~g}, 0.27 \mathrm{mmol})$ in $\mathrm{CH}_{2} \mathrm{Cl}_{2}(15 \mathrm{~mL})$ was added propargyl alcohol complex 6a $(0.100 \mathrm{~g}, 0.292 \mathrm{mmol}) . \mathrm{BF}_{3}-\mathrm{OEt}_{2}(101 \mu \mathrm{L}, 0.797 \mathrm{mmol})$ was added dropwise at $0^{\circ}$ C. After $3 \mathrm{~h}$ of continuous stirring, $\mathrm{NH}_{4} \mathrm{Cl}_{\text {(aq) }}$ was added and the mixture was subjected to a conventional 
extractive workup $\left(\mathrm{CH}_{2} \mathrm{Cl}_{2}\right)$. The residue was subjected to flash chromatography (50:1 petroleum ether: $\left.\mathrm{Et}_{2} \mathrm{O}\right)$ to give 7a $(0.127 \mathrm{~g}, 93 \%)$ : IR $(\mathrm{KBr})_{\max } 3003,2960,2090,2055 \mathrm{~cm}^{-1} ;{ }^{1} \mathrm{H}$ NMR $7.71(\mathrm{~d}, \mathrm{~J}=8.9$, $1 \mathrm{H}), 7.70(\mathrm{~d}, \mathrm{~J}=8.9,1 \mathrm{H}), 7.22(\mathrm{~d}, \mathrm{~J}=2.4,1 \mathrm{H}), 7.10(\mathrm{~d}, \mathrm{~J}=8.9,1 \mathrm{H}), 7.04(\mathrm{dd}, \mathrm{J}=8.9,2.4,1 \mathrm{H}), 5.95(\mathrm{~s}$, 1H), 4.60 (s, 2H), 3.97 (s, 3H), 3.94 (s, 3H); ${ }^{13} \mathrm{C}$ NMR 199.9, 158.4, 154.7, 133.9, 130.2, 128.6, 124.6, $120.1,116.1,109.7,101.6,96.1,73.5,55.4,55.1,29.5 ; \mathrm{MS} 484\left(\mathrm{M}^{+}-\mathrm{CO}\right), 456\left(\mathrm{M}^{+}-2 \mathrm{CO}\right), 428\left(\mathrm{M}^{+}-\right.$ 3CO), $372\left(\mathrm{M}^{+}-5 \mathrm{CO}\right)$; HRMS m/e for $\mathrm{C}_{21} \mathrm{H}_{14} \mathrm{Co}_{2} \mathrm{O}_{8}$ calcd $\left(\mathrm{M}^{+}-2 \mathrm{CO}\right) 455.9454$, found 455.9441 .

\section{Hexacarbonyl[ $\mu$ - 4-(2,7-dimethoxy-1-(3-carbomethoxyprop-2-ynyl)naphthalene)]dicobalt (7b):}

Subjecting 5a $(0.210 \mathrm{~g}, 1.12 \mathrm{mmol})$, 6a $(0.508 \mathrm{~g}, 1.23 \mathrm{mmol})$ and $\mathrm{BF}_{3}-\mathrm{OEt}_{2}(425 \mu \mathrm{L}, 3.35 \mathrm{mmol})$ to Method 1 gave product $7 \mathbf{b}(0.560 \mathrm{~g},, 88 \%$ yield $)$ following flash chromatography (5:1 petroleum ether: $\left.\mathrm{Et}_{2} \mathrm{O}\right)$ as red brown solid: IR $(\mathrm{KBr}){ }_{\max } 3003,2951,2097,2063,2028,1708 ;{ }^{1} \mathrm{H}$ NMR $7.72(\mathrm{~d}, \mathrm{~J}=8.9$, $1 \mathrm{H}), 7.68(\mathrm{~d}, \mathrm{~J}=8.9,1 \mathrm{H}), 7.18(\mathrm{~d}, \mathrm{~J}=2.3,1 \mathrm{H}), 7.10(\mathrm{~d}, \mathrm{~J}=8.9,1 \mathrm{H}), 7.02(\mathrm{dd}, \mathrm{J}=8.9,2.3,1 \mathrm{H}), 4.64(\mathrm{~s}$, 2H), 3.96 (s, 3H), 3.95 (s, 3H), 3.53(s, 3H); ${ }^{13} \mathrm{C}$ NMR 198.4, 170.7, 158.5, 154.7, 133.9, 130.2, 128.8, $124.4,119.1,116.2,109.4,101.1,99.4,79.3,55.2,54.9,52.4,29.1 ; \mathrm{MS}$ m/e $570\left(\mathrm{M}^{+}\right), 542\left(\mathrm{M}^{+}-1 \mathrm{CO}\right)$, $514\left(\mathrm{M}^{+}-2 \mathrm{CO}\right), 486\left(\mathrm{M}^{+}-3 \mathrm{CO}\right), 458\left(\mathrm{M}^{+}-4 \mathrm{CO}\right), 430\left(\mathrm{M}^{+}-5 \mathrm{CO}\right), 402\left(\mathrm{M}^{+}-6 \mathrm{CO}\right) ; \mathrm{HRMS} \mathrm{m} / \mathrm{e}$ for $\mathrm{C}_{23} \mathrm{H}_{16} \mathrm{Co}_{2} \mathrm{O}_{10}$ calcd ( $\left.\mathrm{M}^{+}-\mathrm{CO}\right) 541.9458$, found 541.9455 .

\section{Hexacarbonyl[ $\mu$ - 4-(2,7-dimethoxy-1-(but-2-ynyl)naphthalene)]dicobalt $\quad(7 c): \quad$ Subjecting $\quad 5 a$} $(0.0960 \mathrm{~g}, 0.511 \mathrm{mmol}), \mathbf{6 c}(0.200 \mathrm{~g}, 0.562 \mathrm{mmol})$ and $\mathrm{BF}_{3}-\mathrm{OEt}_{2}(196 \mu \mathrm{L}, 1.55 \mathrm{mmol})$ to Method 1 gave product 7c (0.225 g, 84\% yield) following flash chromatography (100:1 petroleum ether: $\left.\mathrm{Et}_{2} \mathrm{O}\right)$ as red brown solid: IR (KBr) ${ }_{\max } 2941,2086,2044,2015,1629 \mathrm{~cm}^{-1} ;{ }^{1} \mathrm{H}$ NMR $7.73(\mathrm{~d}, \mathrm{~J}=9.0,1 \mathrm{H}), 7.71$ (d, $\mathrm{J}=9.0,1 \mathrm{H}), 7.26(\mathrm{~d}, \mathrm{~J}=2.2,1 \mathrm{H}), 7.12(\mathrm{~d}, \mathrm{~J}=8.9,1 \mathrm{H}), 7.03(\mathrm{dd}, \mathrm{J}=8.9,2.2,1 \mathrm{H}), 4.61(\mathrm{~s}, 2 \mathrm{H}), 3.98(\mathrm{~s}$, 3H), 3.96 (s, 3H), 2.42 (s, 3H); ${ }^{13} \mathrm{C}$ NMR 200.1, 158.4, 154.7, 133.8, 130.2, 128.5, 124.5, 119.6, 116.1, 109.5, 101.6, 98.1, 94.2, 55.3, 55.0, 29.5, 21.0; MS m/e $442\left(\mathrm{M}^{+}-3 \mathrm{CO}\right), 414\left(\mathrm{M}^{+}-4 \mathrm{CO}\right), 386\left(\mathrm{M}^{+}-5 \mathrm{CO}\right)$, $358\left(\mathrm{M}^{+}-6 \mathrm{CO}\right)$; HRMS m/e for $\mathrm{C}_{22} \mathrm{H}_{16} \mathrm{Co}_{2} \mathrm{O}_{8}$ calcd $\left(\mathrm{M}^{+}-\mathrm{CO}\right)$ 497.9560, found 497.9583.

\section{Hexacarbonyl[ $\mu$ - 4-(2,7-dimethoxy-1-(3-trimethylsilylprop-2-ynyl)naphthalene)]dicobalt (7d):}

Subjecting $5 \mathbf{a}(0.0433 \mathrm{~g}, 0.230 \mathrm{mmol}), \mathbf{6 d}(0.1092 \mathrm{~g}, 0.264 \mathrm{mmol})$ and $\mathrm{BF}_{3}-\mathrm{OEt}_{2}(87 \mu \mathrm{L}, 0.69 \mathrm{mmol})$ to 
Method 1 gave product 7d $(0.1308 \mathrm{~g}$, 97\% yield) following flash chromatography (50:1 petroleum ether: $\left.\mathrm{Et}_{2} \mathrm{O}\right)$ as red brown solid: IR $(\mathrm{KBr})_{\max } 2959,2083,2042,2013,1629 \mathrm{~cm}^{-1}$; ${ }^{1} \mathrm{H} \mathrm{NMR} 7.73$ (d, J $=8.8,1 \mathrm{H}), 7.72(\mathrm{~d}, \mathrm{~J}=8.8,1 \mathrm{H}), 7.26(\mathrm{~d}, \mathrm{~J}=2.3,1 \mathrm{H}), 7.12(\mathrm{~d}, \mathrm{~J}=8.9,1 \mathrm{H}), 7.05(\mathrm{dd}, \mathrm{J}=8.9,2.3,1 \mathrm{H})$, 4.72 (br s, 2H), 3.96 (s, 3H), 3.95 (s, 3H), 0.06 (s, 9H); ${ }^{13} \mathrm{C}$ NMR 200.6, 158.7, 154.8, 134.0, 130.3, $128.7,124.8,120.0,115.8,110.6,109.8,102.5,79.3,55.3,55.2,30.6,0.3 ; \mathrm{MS}$ m/e $556\left(\mathrm{M}^{+}-\mathrm{CO}\right), 528$ (M+2CO), $500\left(\mathrm{M}^{+}-3 \mathrm{CO}\right), 444\left(\mathrm{M}^{+}-5 \mathrm{CO}\right)$; HRMS for $\mathrm{C}_{24} \mathrm{H}_{22} \mathrm{Co}_{2} \mathrm{O}_{8} \mathrm{Si}$ calcd. $\left(\mathrm{M}^{+}-3 \mathrm{CO}\right) 499.9900$, found 499.9898.

Hexacarbonyl[ $\mu$ - 4-(2,7-dibenzyloxy-1-(3-carbomethoxyprop-2-ynyl)naphthalene)]dicobalt (7g) and Dodecacarbonyl[ $\mu$ - 4-(2,7-dibenzyloxy-1,8-di(3-carbomethoxyprop-2ynyl)naphthalene)]tetracobalt (9g): Subjecting 2,7-dibenzyloxynaphthalene (5b) (0.754 g, 2.21 mmol), 6b $(1.009 \mathrm{~g}, 2.437 \mathrm{mmol})$ and $\mathrm{BF}_{3}-\mathrm{OEt}_{2}(842 \mu \mathrm{L}, 6.64 \mathrm{mmol})$ to Method 1 gave, in order of elution, $7 \mathrm{~g}$ (1.133g, 71\% yield), and 9g (0.3210 g, 13\% yield), following flash chromatography (5:1 petroleum ether: $\mathrm{Et}_{2} \mathrm{O}$ ) as red brown solid. (7g): IR (KBr) ${ }_{\max } 3039,2957,2098,2031,1706 \mathrm{~cm}^{-1} ;{ }^{1} \mathrm{H}$ NMR $7.81(\mathrm{dd}, \mathrm{J}=8.9,2.4,2 \mathrm{H}), 7.66(\mathrm{~d}, \mathrm{~J}=7.1,2 \mathrm{H}), 7.62(\mathrm{~d}, \mathrm{~J}=7.1,2 \mathrm{H}), 7.45-7.59(\mathrm{~m}, 7 \mathrm{H}), 7.28$ $(\mathrm{dd}, \mathrm{J}=8.7,1.9,1 \mathrm{H}), 7.26(\mathrm{~d}, \mathrm{~J}=8.8,1 \mathrm{H}), 5.39(\mathrm{~s}, 2 \mathrm{H}), 5.33(\mathrm{~s}, 2 \mathrm{H}), 4.81($ br s, $2 \mathrm{H}), 3.56(\mathrm{~s}, 3 \mathrm{H}) ;{ }^{13} \mathrm{C}$ NMR 198.3, 170.5, 157.7, 154.1, 137.0, 134.0, 130.2, 128.8, 128.5, 127.9, 127.6, 127.4, 124.8, 120.0, 116.5, 111.5, 103.1, 99.0, 79.6, 70.7, 69.6, 52.4, 29.5; MS m/e 610 (M+4CO), $554\left(\mathrm{M}^{+}-6 \mathrm{CO}\right)$; HRMS for $\mathrm{C}_{35} \mathrm{H}_{24} \mathrm{Co}_{2} \mathrm{O}_{10}$ calcd. ( $\left.\mathrm{M}^{+}-6 \mathrm{CO}\right)$ 554.0339, found 554.0354.

(9g): IR (KBr) $\max _{\text {max }} 2951,2097,2063,2030,1709 \mathrm{~cm}^{-1} ; 7.67(\mathrm{~d}, \mathrm{~J}=8.9,2 \mathrm{H}), 7.51(\mathrm{~d}, \mathrm{~J}=7.3,4 \mathrm{H})$, 7.40 (apparent t, J = 7.3, 4H), $7.33(\mathrm{t}, \mathrm{J}=7.1,2 \mathrm{H}), 7.16(\mathrm{~d}, \mathrm{~J}=8.9,2 \mathrm{H}), 5.35(\mathrm{~d}, \mathrm{~J}=16.7,2 \mathrm{H}), 5.30(1 / 2$ ABquartet, $\mathrm{J}=12.2,2 \mathrm{H}), 5.22(1 / 2$ ABquartet, $\mathrm{J}=12.2,2 \mathrm{H}), 4.82(\mathrm{~d}, \mathrm{~J}=16.6,2 \mathrm{H}), 3.36(\mathrm{~s}, 6 \mathrm{H}) ;{ }^{13} \mathrm{C}$ NMR 198.2, 170.4, 155.6, 137.0, 132.2, 130.8, 128.5, 127.9, 127.2, 126.8, 121.4, 112.0, 99.6, 80.2, 71.0, 52.3, 31.0; MS m/e ; Anal. Calc. For $\mathrm{C}_{46} \mathrm{H}_{28} \mathrm{Co}_{4} \mathrm{O}_{18}$ : C, 50.02; H, 2.56. Found C, 49.87, H, 2.52.

\section{Hexacarbonyl[ $\mu$ - 4-(2,7-dimethoxy-1-(1-methylprop-2-ynyl)naphthalene)]dicobalt}

(7e):

Subjecting 5a $(0.0498 \mathrm{~g}, 0.264 \mathrm{mmol}), \mathbf{6 e}(0.0880 \mathrm{~g}, 0.247 \mathrm{mmol})$ and $\mathrm{BF}_{3}-\mathrm{OEt}_{2}(105 \mu \mathrm{L}, 0.829 \mathrm{mmol})$ to Method 1 gave product 7e $(0.0920 \mathrm{~g}, 66 \%$ yield $)$ following flash chromatography (40:1 petroleum ether: 
$\left.\mathrm{Et}_{2} \mathrm{O}\right)$ as red brown solid: IR $(\mathrm{KBr})_{\max } 3039,2957,2098,2031,1706 \mathrm{~cm}^{-1} ;{ }^{1} \mathrm{H}$ NMR $(58: 42$ mixture of rotamers) $7.66-7.70(\mathrm{~m}, 2 \mathrm{H}$, both), $7.60(\mathrm{~d}, \mathrm{~J}=2.3,1 \mathrm{H}$ minor $), 7.35(\mathrm{~d}, \mathrm{~J}=1.9,1 \mathrm{H}$ major $), 7.10(\mathrm{~d}, \mathrm{~J}=$ 8.9, 1H, minor), 7.08 (d, J = 8.8, 1H, major), 7.03 (dd, J = 8.8, 2.3, 1H, major), 7.02 (dd, $\mathrm{J}=8.9,1.9,1 \mathrm{H}$ minor), 6.09 (s, 1H, both), 5.65 (q, J = 7.5, 1H, minor), 5.04 (q, J = 6.9, 1H, major), 3.99 (s, 3H, minor), 3.97 (s, 3H, major), $3.96(\mathrm{~s}, 3 \mathrm{H}$, minor), 3.93 (s, 3H, major), 1.89 (d, J = 6.9, 3H, major), $1.88(\mathrm{~d}, \mathrm{~J}=$ 7.5, 3H, minor); ${ }^{13} \mathrm{C}$ NMR 200.3, 199.9, 158.7, 157.1, 156.3, 154.1, 133.5, 133.3, 130.6, 130.4, 128.9, $128.8,125.4,124.9,124.7,124.4,116.1,115.5,111.0,110.3,104.6,102.4,101.8,101.0,74.1,72.6$ 56.1, 55.3, 55.2, 55.0, 37.7, 35.3, 22.4, 21.5; MS m/e $498\left(\mathrm{M}^{+}-\mathrm{CO}\right), 470\left(\mathrm{M}^{+}-2 \mathrm{CO}\right), 442\left(\mathrm{M}^{+}-3 \mathrm{CO}\right), 414$ ( $\left.\mathrm{M}^{+}-4 \mathrm{CO}\right), 386\left(\mathrm{M}^{+}-5 \mathrm{CO}\right)$; HRMS m/e for $\mathrm{C}_{22} \mathrm{H}_{16} \mathrm{Co}_{2} \mathrm{O}_{8}$ calc. $\left(\mathrm{M}^{+}-2 \mathrm{CO}\right) 469.9611$, found 469.9608.

\section{Hexacarbonyl[ $\mu$ - 4-(2,7-dimethoxy-3-(1-phenylprop-2-ynyl)naphthalene)]dicobalt}

(8f):

Subjecting 5a $(0.0560 \mathrm{~g}, 0.298 \mathrm{mmol}), \mathbf{6 f}(0.1500 \mathrm{~g}, 0.3588 \mathrm{mmol})$ and $\mathrm{BF}_{3}-\mathrm{OEt}_{2}(113 \mu \mathrm{L}, 0.892 \mathrm{mmol})$ to Method 1 gave product $\mathbf{8 f}(0.0900 \mathrm{~g}, 51 \%$ yield $)$ following flash chromatography (10:1 petroleum ether: $\left.\mathrm{Et}_{2} \mathrm{O}\right)$, as red brown solid; $(\mathbf{8 f}) \mathrm{IR}(\mathrm{KBr}){ }_{\max } 2922,2089,2050,2014,1632 \mathrm{~cm}^{-1} ;{ }^{1} \mathrm{H}$ NMR 7.78 $(\mathrm{s}, 1 \mathrm{H}), 7.62(\mathrm{~d}, \mathrm{~J}=8.9,1 \mathrm{H}), 7.48(\mathrm{~d}, \mathrm{~J}=7.3,2 \mathrm{H}), 7.26(\mathrm{~m}, 2 \mathrm{H}), 7.18(\mathrm{t}, \mathrm{J}=7.3,1 \mathrm{H}), 7.02$ (obscured d, $\mathrm{J}=2.5,1 \mathrm{H}), 7.01(\mathrm{~s}, 1 \mathrm{H}), 6.98(\mathrm{dd}, \mathrm{J}=8.9,2.5,1 \mathrm{H}), 6.46(\mathrm{~s}, 1 \mathrm{H}), 6.01(\mathrm{~s}, 1 \mathrm{H}), 3.93(\mathrm{~s}, 3 \mathrm{H}), 3.89(\mathrm{~s}$, $3 \mathrm{H}) ;{ }^{13} \mathrm{C}$ NMR 199.5, 158.1, 155.5, 144.2, 134.9, 131.4, 129.1, 128.4, 127.9, 126.9, 123.8, 116.2, 105.0, 104.8, 100.5, 74.1, 55.4, 55.2, 48.7; MS m/e $532\left(\mathrm{M}^{+}-2 \mathrm{CO}\right), 476\left(\mathrm{M}^{+}-4 \mathrm{CO}\right), 448\left(\mathrm{M}^{+}-5 \mathrm{CO}\right), 420\left(\mathrm{M}^{+}-\right.$ 6CO); HRMS m/e for $\mathrm{C}_{27} \mathrm{H}_{18} \mathrm{Co}_{2} \mathrm{O}_{8}$ calcd. $\left(\mathrm{M}^{+}-4 \mathrm{CO}\right) 475.9869$, found 475.9853.

\section{Dodecacarbonyl $[\mu$ - 4-(2,7-dimethoxy-1,8-di(prop-2-ynyl)naphthalene)]tetracobalt $\quad(9 a) \quad$ and} Dodecacarbonyl[ $\mu$ - 4-(2,7-dimethoxy-1,6-di(prop-2-ynyl)naphthalene)]tetracobalt (10a). Method 2.: To a solution of 2,7-dimethoxynaphthalene $\mathbf{5 a}(0.100 \mathrm{~g}, 0.531 \mathrm{mmol})$ in $\mathrm{CH}_{2} \mathrm{Cl}_{2}(10 \mathrm{~mL})$ was added propargyl alcohol complex $6 \mathbf{a}(0.393 \mathrm{~g}, 1.149 \mathrm{mmol})$, followed by the dropwise addition of $\mathrm{BF}_{3}-\mathrm{OEt}_{2}$ $(202 \mu \mathrm{L}, 1.59 \mathrm{mmol})$ at $0^{\circ} \mathrm{C}$. After $3 \mathrm{~h}$ of continuous stirring, $\mathrm{NH}_{4} \mathrm{Cl}_{\text {(aq) }}$ was added and the mixture was subjected to a conventional extractive workup. The residue was subjected purified by flash chromatography (10:1 petroleum ether: $\left.\mathrm{Et}_{2} \mathrm{O}\right)$ to give, in order of elution, $10 \mathbf{a}(0.040 \mathrm{~g}, 9 \%$ yield $)$, and 9a $\left(0.280 \mathrm{~g}, 63 \%\right.$ yield); (9a): IR $(\mathrm{KBr}){ }_{\max } 2917,2090,2051,2021 \mathrm{~cm}^{-1} ;{ }^{1} \mathrm{H}$ NMR $7.70(\mathrm{~d}, \mathrm{~J}=8.9$, 
$2 \mathrm{H}), 7.12(\mathrm{~d}, \mathrm{~J}=8.9,2 \mathrm{H}), 5.83(\mathrm{~s}, 2 \mathrm{H}), 5.22(\mathrm{~d}, \mathrm{~J}=16.4,2 \mathrm{H}), 4.50(\mathrm{~d}, \mathrm{~J}=16.4,2 \mathrm{H}), 3.99(\mathrm{~s}, 6 \mathrm{H}) ;{ }^{13} \mathrm{C}$ NMR 199.6, 156.1, 131.9, 130.7, 126.5, 121.3, 110.1, 97.5, 73.8, 55.9, 30.6; MS m/e $808\left(\mathrm{M}^{+}-1 \mathrm{CO}\right)$,


8CO); HRMS m/e for $\mathrm{C}_{30} \mathrm{H}_{16} \mathrm{Co}_{4} \mathrm{O}_{14}$ calcd. $\left(\mathrm{M}^{+}-\mathrm{CO}\right)$ 807.7917, found 807.7904.

(10a): IR (KBr) ${ }_{\max } 2922,2091,2048,2016 \mathrm{~cm}^{-1} ;{ }^{1} \mathrm{H}$ NMR 7.67 (d, J = 8.5, 1H), 7.57 (s, 1H), 7.19 (s, 1H), $7.08(\mathrm{~d}, \mathrm{~J}=8.5,1 \mathrm{H}), 6.05(\mathrm{~s}, 1 \mathrm{H}), 5.86(\mathrm{~s}, 1 \mathrm{H}), 4.59(\mathrm{~s}, 2 \mathrm{H}), 4.26(\mathrm{~s}, 2 \mathrm{H}), 3.97(\mathrm{~s}, 3 \mathrm{H}), 3.96(\mathrm{~s}$, $3 \mathrm{H}) ;{ }^{13} \mathrm{C} 199.8,156.5,154.4,133.2,130.4,128.2,127.8,124.4,120.0,110.0,100.9,97.9,96.1,73.8$, 73.4, 55.5, 54.7, 39.3 , 29.7 ; MS m/e $640\left(\mathrm{M}^{+}-6 \mathrm{CO}\right), 612\left(\mathrm{M}^{+}-7 \mathrm{CO}\right), 584\left(\mathrm{M}^{+}-8 \mathrm{CO}\right), 500\left(\mathrm{M}^{+}-12 \mathrm{CO}\right)$; HRMS m/e for $\mathrm{C}_{30} \mathrm{H}_{16} \mathrm{Co}_{4} \mathrm{O}_{14}$ calcd. (M+7CO) 639.8224, found 639.8223.

\section{Dodecacarbonyl[ $\mu$ - 4-(2,7-dimethoxy-1,8-di(3-carbomethoxyprop-2-}

ynyl)naphthalene)]tetracobalt (9b): Subjecting a solution of 2,7-dimethoxynaphthalene 5a (0.0258 g, $0.137 \mathrm{mmol})$, propargyl ether complex $\mathbf{6 b}(0.1314 \mathrm{~g}, 0.317 \mathrm{mmol})$ and $\mathrm{BF}_{3}-\mathrm{OEt}_{2}(115 \mu \mathrm{L}, 0.91 \mathrm{mmol})$ to Method 2 gave $9 b(0.1117 \mathrm{~g}, 86 \%)$ following g flash chromatography $\left(5: 1\right.$ petroleum ether: $\left.\mathrm{Et}_{2} \mathrm{O}\right)$ as red brown solid: IR (KBr) $\quad{ }_{\max } 3004,2950,2097,2067,1710 \mathrm{~cm}^{-1} ;{ }^{1} \mathrm{H}$ NMR $7.71(\mathrm{~d}, \mathrm{~J}=9.0,2 \mathrm{H}), 7.10$ $(\mathrm{d}, \mathrm{J}=9.0,2 \mathrm{H}), 5.19(\mathrm{~d}, \mathrm{~J}=16.5,2 \mathrm{H}), 4.69(\mathrm{~d}, \mathrm{~J}=16.5,2 \mathrm{H}), 3.94(\mathrm{~s}, 6 \mathrm{H}), 3.46(\mathrm{~s}, 6 \mathrm{H}) ;{ }^{13} \mathrm{C}$ NMR 198.2, $170.5,156.0,132.0,130.9,126.3,120.2,109.7,99.8,80.2,55.6,52.3,30.8 ; \mathrm{MS} \mathrm{m} / \mathrm{e} 896\left(\mathrm{M}^{+}-2 \mathrm{CO}\right), 840$ (M+4CO), $784\left(\mathrm{M}^{+}-6 \mathrm{CO}\right), 728\left(\mathrm{M}^{+}-8 \mathrm{CO}\right), 700\left(\mathrm{M}^{+}-9 \mathrm{CO}\right)$; Anal. for $\mathrm{C}_{34} \mathrm{H}_{20} \mathrm{Co}_{4} \mathrm{O}_{18}$ : calcd. C, 42.88; H, 2.12. Found: C, 43.12, H, 2.10.

\section{Dodecacarbonyl[ $\mu$ - 4-(2,7-dimethoxy-1,6-di(but-2-ynyl)naphthalene)]tetracobalt}

(10c).

Subjecting 5a $(0.1000 \mathrm{~g}, 0.5314 \mathrm{mmol}), \mathbf{6 c}(0.400 \mathrm{~g}, 1.123 \mathrm{mmol})$ and $\mathrm{BF}_{3}-\mathrm{OEt}_{2}(202 \mu \mathrm{L}, 1.59 \mathrm{mmol})$ to Method 2 to give product 10c $(0.3950 \mathrm{~g}, 86 \%$ yield) following flash chromatography (100:1 petroleum ether: $\left.\mathrm{Et}_{2} \mathrm{O}\right)$ as red brown solid: IR $(\mathrm{KBr})_{\text {max }} 2949,2085,2000,1631 \mathrm{~cm}^{-1} ;{ }^{1} \mathrm{H}$ NMR $7.71(\mathrm{~d}, \mathrm{~J}=8.9$, 1H), $7.60(\mathrm{~s}, 1 \mathrm{H}), 7.27(\mathrm{~s}, 1 \mathrm{H}), 7.13(\mathrm{~d}, \mathrm{~J}=8.9,1 \mathrm{H}), 4.62(\mathrm{~s}, 2 \mathrm{H}), 4.30(\mathrm{~s}, 2 \mathrm{H}), 4.01(\mathrm{~s}, 3 \mathrm{H}), 3.98(\mathrm{~s}$, 3H), 2.55 (s, 3H), 2.44 (s, 3H); ${ }^{13} \mathrm{C}$ NMR 200.1, 156.6, 154.6, 133.3, 130.4, 128.3, 127.4, 124.4, 119.6, 109.8, 100.7, 99.3, 98.2, 94.3, 55.3, 54.7, 34.8, 29.7, 21.0, 20.4; MS m/e 808 ( $\left.\mathrm{M}^{+}-2 \mathrm{CO}\right), 780$ (M+3CO), 
$724\left(\mathrm{M}^{+}-5 \mathrm{CO}\right), 640\left(\mathrm{M}^{+}-8 \mathrm{CO}\right), 612\left(\mathrm{M}^{+}-9 \mathrm{CO}\right)$; HRMS m/e for $\mathrm{C}_{32} \mathrm{H}_{20} \mathrm{Co}_{4} \mathrm{O}_{14}$, calcd $\left(\mathrm{M}^{+}-3 \mathrm{CO}\right) 779.8334$, found 779.8342 .

\section{Dodecacarbonyl[ $\mu$ - 4-(2,7-dimethoxy-1,6-di(3-trimethylsilylprop-2-}

ynyl)naphthalene)]tetracobalt (10d): Subjecting 5a $(0.050 \mathrm{~g}, 0.266 \mathrm{mmol}), \mathbf{6 d}(0.242 \mathrm{~g}, 0.585 \mathrm{mmol})$ and $\mathrm{BF}_{3}-\mathrm{OEt}_{2}(118 \mu \mathrm{L}, 0.931 \mathrm{mmol})$ to Method 2, with the exception that the reaction mixture was additionally warmed to room temperature for $5 \mathrm{~h}$. Flash chromatography $\left(50: 1\right.$ petroleum ether: $\left.\mathrm{Et}_{2} \mathrm{O}\right)$ gave, in order of elution, $\mathbf{1 0 d}(0.105 \mathrm{~g}, 40 \%$ yield $)$ as a red-brown solid, and $\mathbf{7 d}(0.091 \mathrm{~g}, 59 \%) ;(\mathbf{1 0 d})$ : IR $(\mathrm{KBr})_{\max } 2960,2089,2050,2023,1632 \mathrm{~cm}^{-1} ;{ }^{1} \mathrm{H}$ NMR $7.70(\mathrm{~d}, \mathrm{~J}=8.9,1 \mathrm{H}), 7.61(\mathrm{~s}, 1 \mathrm{H}), 7.21(\mathrm{~s}$, 1H), $7.12(\mathrm{~d}, \mathrm{~J}=8.9,1 \mathrm{H}), 4.70$ (br s, 2H), $4.38(\mathrm{~s}, 2 \mathrm{H}), 3.97(\mathrm{~s}, 3 \mathrm{H}), 3.94(\mathrm{~s}, 3 \mathrm{H}), 0.25$ (s, 9H), $0.12(\mathrm{~s}$, 9H); ${ }^{13} \mathrm{C}$ NMR 200.3., 156.6, 154.9, 133.4, 131.1, 128.3, 127.6, 124.5, 119.9, 112.7, 110.6, 110.0, 100.7, 79.4,79.2, 55.3, 54.6, 34.9, 30.7, 0.5, 0.4; MS m/e $952\left(\mathrm{M}^{+}-\mathrm{CO}\right), 924\left(\mathrm{M}^{+}-2 \mathrm{CO}\right), 896\left(\mathrm{M}^{+}-3 \mathrm{CO}\right), 840$

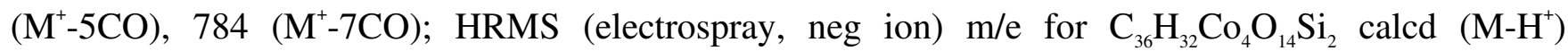
978.8580; found 978.8584.

\section{Dodecacarbonyl[ $\mu$ - 4-(6-(but-3-yn-2-yl)-2,7-dimethoxy-1-(prop-2-ynyl)naphthalene)]tetracobalt}

(10e): Subjecting 7a $(0.0300 \mathrm{~g}, 0.0585 \mathrm{mmol}), \mathbf{6 e}(0.0330 \mathrm{~g}, 0.0926 \mathrm{mmol})$ and $\mathrm{BF}_{3}-\mathrm{OEt}_{2}(25 \mu \mathrm{L}, 0.20$ mmol) to Method 1 to give product 10e $(0.0360 \mathrm{~g}, 72 \%$ yield $)$ following flash chromatography (100:1 petroleum ether: $\left.\mathrm{Et}_{2} \mathrm{O}\right)$ as red brown solid; (10e): IR (KBr) ${ }_{\max } 2935,2089,2050,2018,1627 \mathrm{~cm}^{-1} ;{ }^{1} \mathrm{H}^{2}$ NMR $7.69(\mathrm{~d}, \mathrm{~J}=8.9,1 \mathrm{H}), 7.63(\mathrm{~s}, 1 \mathrm{H}), 7.22(\mathrm{~s}, 1 \mathrm{H}), 7.09(\mathrm{~d}, \mathrm{~J}=8.9,1 \mathrm{H}), 6.02(\mathrm{~s}, 1 \mathrm{H}), 5.90(\mathrm{~s}, 1 \mathrm{H})$, $4.80(\mathrm{q}, \mathrm{J}=7.1,1 \mathrm{H}), 4.66(1 / 2$ ABquartet, $\mathrm{J}=15.2,1 \mathrm{H}), 4.57(1 / 2$ ABquartet, $\mathrm{J}=15.3,1 \mathrm{H}), 4.01(\mathrm{~s}$, 3H), 3.97 (s, 3H), 1.77 (d, J = 7.1, 3H); ${ }^{13} \mathrm{C}$ NMR 199.9, 156.1, 154.5, 133.0, 132.8, 128.5, 126.8, 124.4, $119.9,110.0,105.4,101.0,96.2,74.1,73.5,55.5,54.9,35.3,30.0,22.1 ; \mathrm{MS}$ m/e $822\left(\mathrm{M}^{+}-\mathrm{CO}\right), 794\left(\mathrm{M}^{+}-\right.$ 2CO), $766\left(\mathrm{M}^{+}-3 \mathrm{CO}\right), 738\left(\mathrm{M}^{+}-4 \mathrm{CO}\right), 710\left(\mathrm{M}^{+}-5 \mathrm{CO}\right), 682\left(\mathrm{M}^{+}-6 \mathrm{CO}\right), 654\left(\mathrm{M}^{+}-7 \mathrm{CO}\right), 626\left(\mathrm{M}^{+}-8 \mathrm{CO}\right)$, 598 ( $\left.\mathrm{M}^{+}-9 \mathrm{CO}\right)$; HRMS m/e for $\mathrm{C}_{31} \mathrm{H}_{18} \mathrm{Co}_{4} \mathrm{O}_{14}$ calcd. $\left(\mathrm{M}^{+}-4 \mathrm{CO}\right) 737.8228$, found 737.8201 .

\section{Dodecacarbonyl[ $\mu$ - 4-(2,7-dimethoxy-6-(1-phenylprop-2-ynyl)-1-(prop-2-ynyl)naphthalene)]}

tetracobalt (10f): Subjecting $\mathbf{8 f}(0.0372 \mathrm{~g}, 0.0632 \mathrm{mmol}), \mathbf{6 a}(0.0238 \mathrm{~g}, 0.0695 \mathrm{mmol})$ and $\mathrm{BF}_{3}-\mathrm{OEt}_{2}$ (24 $\mu \mathrm{L}, 0.19 \mathrm{mmol})$ to Method 1 to give product $\mathbf{1 0 f}(0.0380 \mathrm{~g}, 65 \%$ yield $)$ following flash 
chromatography (10:1 petroleum ether: $\left.\mathrm{Et}_{2} \mathrm{O}\right)$ as red brown solid; (10f) IR (KBr) ${ }_{\max }$ 2929, 2089, 2049, 2018, $1629 \mathrm{~cm}^{-1} ;{ }^{1} \mathrm{H}$ NMR $7.80(\mathrm{~s}, 1 \mathrm{H}), 7.66(\mathrm{~d}, \mathrm{~J}=9.0,1 \mathrm{H}), 7.51(\mathrm{~d}, \mathrm{~J}=7.6,2 \mathrm{H}), 7.27-7.31$ (apparent $\mathrm{t}, \mathrm{J}=7.6,2 \mathrm{H}), 7.21(\mathrm{t}, \mathrm{J}=7.4,1 \mathrm{H}), 7.17(\mathrm{~d}, \mathrm{~J}=1.0,1 \mathrm{H}), 7.07(\mathrm{~d}, \mathrm{~J}=9.0,1 \mathrm{H}), 6.46(\mathrm{~s}, 1 \mathrm{H}), 6.05(\mathrm{~s}, 1 \mathrm{H})$, $5.88(\mathrm{~s}, 1 \mathrm{H}), 4.60(1 / 2 \mathrm{AB}$ quartet, $\mathrm{J}=15.4,1 \mathrm{H}), 4.52$ (1/2 ABquartet, $\mathrm{J}=15.4,1 \mathrm{H}), 3.97(\mathrm{~s}, 3 \mathrm{H}), 3.95$ (s, 3H); ${ }^{13} \mathrm{C}$ NMR 199.4, 155.7, 154.5, 144.0, 132.8, 131.4, 128.9, 128.6, 128.4, 126.9, 124.2, 119.8, 110.0, 101.1, 100.4, 96.0, 74.0, 73.5, 55.4, 55.2, 48.5, 29.6; MS m/e $884\left(\mathrm{M}^{+}-\mathrm{CO}\right), 856\left(\mathrm{M}^{+}-2 \mathrm{CO}\right), 828$ ( $\left.\mathrm{M}^{+}-3 \mathrm{CO}\right), 800\left(\mathrm{M}^{+}-4 \mathrm{CO}\right)$; HRMS for $\mathrm{C}_{36} \mathrm{H}_{20} \mathrm{Co}_{4} \mathrm{O}_{14}$, calcd. $\left(\mathrm{M}^{+}-4 \mathrm{CO}\right) 799.8384$, found 799.8414 .

2,7-Dimethoxy-1,8-di(prop-2-ynyl)naphthalene (11): To a solution of 9a (0.0970 g, $0.116 \mathrm{mmol})$ in acetone $(30 \mathrm{~mL})$ with silica gel $(0.600 \mathrm{~g})$ was added ceric ammonium nitrate $(0.360 \mathrm{~g})$ at $-78^{\circ} \mathrm{C}$. The reaction was stirred for $4 \mathrm{~h}$, followed by the addition of $\mathrm{H}_{2} \mathrm{O}$ and filtration through Celite ${ }^{\circledR}$. After a conventional aqueous workup, the mixture was filtered through Celite ${ }^{\circledR}$ and concentrated under reduced pressure. Preparative TLC (10:1 petroleum ether : $\left.\mathrm{Et}_{2} \mathrm{O}\right)$ afforded, in order of elution, 9a $(0.0350 \mathrm{~g}, 36 \%$ recovery), and 11 (0.0175 g, 57\%, 89\% based on recovered starting material); (11): $\mathrm{mp} 144-145{ }^{\circ} \mathrm{C}$; IR $(\mathrm{KBr})_{\max } 3291,2932,2107,1618 \mathrm{~cm}^{-1} ;{ }^{1} \mathrm{H}$ NMR $7.72(\mathrm{~d}, \mathrm{~J}=9.0,2 \mathrm{H}), 7.18(\mathrm{~d}, \mathrm{~J}=9.0,2 \mathrm{H}), 4.31(\mathrm{~d}, \mathrm{~J}$ $=2.6,4 \mathrm{H}), 4.03(\mathrm{~s}, 6 \mathrm{H}), 2.16(\mathrm{t}, \mathrm{J}=2.6,2 \mathrm{H}) ;{ }^{13} \mathrm{C} \mathrm{NMR} 156.7,133.5,130.1,126.2,117.3,111.0,84.7$, 69.1, 56.9, 17.3; MS m/e $264\left(\mathrm{M}^{+}\right)$; HRMS m/e for $\mathrm{C}_{18} \mathrm{H}_{16} \mathrm{O}_{2}$, calcd $\left(\mathrm{M}^{+}\right)$264.1150, found 264.1153.

1,8-Diallyl-2,7-dimethoxynaphthalene (12): To a solution of $11(.0200 \mathrm{~g}, 0.0758 \mathrm{mmol})$ in $20 \mathrm{~mL}$ mixture of ethyl acetate: 1-hexene: pyridine (10:1:1), Lindlar catalyst added (5 mole \%) at room temperature. The reaction was stirred under an $\mathrm{H}_{2}$ atmosphere for $3 \mathrm{~h}$. The reaction mixture was filtered through Celite ${ }^{\circledR}$, and the filtrate was concentrated under reduced pressure. The residue was purified by preparative TLC (5:1 petroleum ether : $\left.\mathrm{Et}_{2} \mathrm{O}\right)$ to give, in order of elution, $12(0.0180 \mathrm{~g}, 0.0671 \mathrm{mmol}$, $89 \%$ yield, $98 \%$ based on recovered starting material), and 11 (0.0018 g); (12): mp 93-94 ${ }^{\circ} \mathrm{C}$; lit. ${ }^{18}$ 189.4-194 ${ }^{\circ} \mathrm{C}$; IR (KBr) ${ }_{\max } 3077,2934,1614 \mathrm{~cm}^{-1} ;{ }^{1} \mathrm{H}$ NMR $7.72(\mathrm{~d}, \mathrm{~J}=8.9,2 \mathrm{H}), 7.18(\mathrm{~d}, \mathrm{~J}=8.9$, 2H), $6.22(\mathrm{~m}, 2 \mathrm{H}), 5.09$ (dd J= 10.3, 1.9, 2H), 4.84 (dd, J= 17.3, 1.9, 2H), 3.93 (s, 6H), 3.91 (br s, 4H); ${ }^{13}$ C NMR 155.7, 139.0, 134.9, 129.4, 126.3, 120.4, 114.5, 111.0, 56.8, 30.9; MS m/e $268\left(\mathrm{M}^{+}\right)$; HRMS m/e for $\mathrm{C}_{18} \mathrm{H}_{20} \mathrm{O}_{2}$ calcd $\left(\mathrm{M}^{+}\right)$268.1463, found 268.1466. 
(Z)-1,6-Dimethoxy-7,10-dihydrocyclohepta[de]naphthalene (13): To a solution of $12(0.0170 \mathrm{~g}$, $0.0634 \mathrm{mmol})$ in $\mathrm{CH}_{2} \mathrm{Cl}_{2}(2 \mathrm{~mL})$, was added Grubbs' 1 Catalyst $(0.0027 \mathrm{~g}, 5 \mathrm{~mole} \%)$ at room temperature. After $6 \mathrm{~h}$, the mixture was filtered through Celite ${ }^{\circledR}$. The filtrate was concentrated under reduced pressure and the crude product was purified by preparative TLC (100:1 petroleum ether: $\left.\mathrm{Et}_{2} \mathrm{O}\right)$ to give 13 (0.0130 g, $0.0541 \mathrm{mmol}, 85 \%)$ : $\mathrm{mp} 95-96{ }^{\circ} \mathrm{C}$; IR $(\mathrm{KBr}){ }_{\max }{ }^{1} \mathrm{H} \mathrm{NMR} \quad 7.58(\mathrm{~d}, \mathrm{~J}=9.0,2 \mathrm{H})$, $7.10(\mathrm{~d}, \mathrm{~J}=9.0,2 \mathrm{H}), 6.19(\mathrm{~m}, 2 \mathrm{H}), 4.02(\mathrm{~d}, \mathrm{~J}=5.6,4 \mathrm{H}), 3.92(\mathrm{~s}, 6 \mathrm{H}) ;{ }^{13} \mathrm{C}$ NMR 154.0, 134.8, 130.9, 128.3, 126.8, 120.2, 112.0, 57.3, 24.3; MS m/e $240\left(\mathrm{M}^{+}\right)$, HRMS m/e for $\mathrm{C}_{16} \mathrm{H}_{16} \mathrm{O}_{2}$ calcd $\left(\mathrm{M}^{+}\right)$240.1150, found 240.1150 .

2,7-Dimethoxy-1-(3-carbomethoxyprop-2-ynyl)naphthalene (14): Method 3. To a solution of 7b $(0.446 \mathrm{~g}, 0.782 \mathrm{mmol})$ in THF $(50 \mathrm{~mL})$ at room temperature, an excess of iodine $\left(\mathrm{I}_{2}\right)$ was added. The solution was stirred for $3 \mathrm{~h}$. Following the addition of aqueous sodium bisulfate, and the mixture subjected to a conventional extractive workup $\left(\mathrm{Et}_{2} \mathrm{O}\right)$. Purification by preparative TLC (1:1 petroleum ether: $\left.\mathrm{Et}_{2} \mathrm{O}\right)$ gave $14\left(0.218 \mathrm{~g}, 98 \%\right.$ yield): $\mathrm{mp} 80-81{ }^{\circ} \mathrm{C}$; IR $(\mathrm{KBr}) \quad{ }_{\max } 2956,2233,1712,1628 ;{ }^{1} \mathrm{H}$ NMR $7.72(\mathrm{~d}, \mathrm{~J}=9.0,1 \mathrm{H}), 7.69(\mathrm{~d}, \mathrm{~J}=8.9,1 \mathrm{H}), 7.22(\mathrm{~d}, \mathrm{~J}=2.4,1 \mathrm{H}), 7.11(\mathrm{~d}, \mathrm{~J}=9.0,1 \mathrm{H}), 7.05(\mathrm{dd}, \mathrm{J}$ $=8.9,2.4,1 \mathrm{H}), 4.11(\mathrm{~s}, 2 \mathrm{H}), 3.97(\mathrm{~s}, 3 \mathrm{H}), 3.96(\mathrm{~s}, 3 \mathrm{H}), 3.70(\mathrm{~s}, 3 \mathrm{H}) ;{ }^{13} \mathrm{C} \mathrm{NMR}$ 158.4, 154.6, 154.0, $133.7,129.9,128.9,124.3,116.1,113.6,110.1,101.2,87.7,71.9,56.1,55.0,52.2,14.5 ;$ MS m/e 284 $\left(\mathrm{M}^{+}\right)$; Anal. for $\mathrm{C}_{17} \mathrm{H}_{16} \mathrm{O}_{4}$ Calcd. C, 71.82; H, 5.67. Found: C, 71.72; H, 5.47.

\section{2,7-Dimethoxy-1-(3-carbomethoxypropyl)naphthalene (15): To a solution of 14 (0.210 g, 0.739} mmol) in $\mathrm{MeOH}(20 \mathrm{~mL})$ under $\mathrm{H}_{2}, \mathrm{Rh} / \mathrm{C}$ (excess) at room temperature. The solution was stirred for $6 \mathrm{~h}$ with monitoring by TLC. The suspension was filtered and the solvent was removed under reduced pressure. Preparative TLC (2:1 petroleum ether: $\left.\mathrm{Et}_{2} \mathrm{O}\right)$ to give 15 ( $0.211 \mathrm{~g}, 99 \%$ yield): bp $170-175{ }^{\circ} \mathrm{C}$ (0.5 torr); IR (KBr) ${ }_{\max } 2954,1740,1628 ;{ }^{1} \mathrm{H}$ NMR $7.69(\mathrm{~d}, \mathrm{~J}=8.9,1 \mathrm{H}), 7.66(\mathrm{~d}, \mathrm{~J}=8.9,1 \mathrm{H}), 7.32(\mathrm{~d}$, $\mathrm{J}=2.0,1 \mathrm{H}), 7.12(\mathrm{~d}, \mathrm{~J}=8.9,1 \mathrm{H}), 7.03(\mathrm{dd}, \mathrm{J}=8.9,2.0,1 \mathrm{H}), 3.98(\mathrm{~s}, 3 \mathrm{H}), 3.93(\mathrm{~s}, 3 \mathrm{H}), 3.70(\mathrm{~s}, 3 \mathrm{H})$, $3.11(\mathrm{t}, \mathrm{J}=7.6,2 \mathrm{H}), 2.44(\mathrm{t}, \mathrm{J}=7.1,2 \mathrm{H}), 2.00(\mathrm{~m}, 2 \mathrm{H}) ;{ }^{13} \mathrm{C}$ NMR 174.1, 158.2, 155.0, 134.3, 129.9, 127.4, 124.7, 121.4, 115.7, 110.5, 101.9, 56.1, 55.1, 51.2, 33.5, 24.4, 24.2; MS m/e $288\left(\mathrm{M}^{+}\right)$; HRMS $\mathrm{m} / \mathrm{e}$ for $\mathrm{C}_{17} \mathrm{H}_{20} \mathrm{O}_{4}$ calcd $\left(\mathrm{M}^{+}\right)$288.1362, found 288.1360. 
1-(4-Hydroxy-4-methylpentyl)-2,7-dimethoxynaphthalene (16): To a solution of 15 (0.114 g, 0.396

$\mathrm{mmol})$ in $\mathrm{Et}_{2} \mathrm{O}(10 \mathrm{~mL})$ at $0{ }^{\circ} \mathrm{C}, \mathrm{MeLi}(1.9 \mathrm{~mL}, 1.5 \mathrm{M}, 2.9 \mathrm{mmol})$ was added. The solution was stirred for $3 \mathrm{~h}$, at which time aqueous $\mathrm{NH}_{4} \mathrm{Cl}$ was added and a conventional extractive workup $\left(\mathrm{Et}_{2} \mathrm{O}\right)$ performed. The volatiles were removed under reduced pressure. Purification by preparative TLC (1:1 petroleum ether: $\left.\mathrm{Et}_{2} \mathrm{O}\right)$ gave 15 (0.103 g, $0.356 \mathrm{mmol}, 90 \%$ yield): mp 45-46 ${ }^{\circ} \mathrm{C}$; IR (KBr) ${ }_{\max } 3422$ br, 2966, $1627 \mathrm{~cm}^{-1}$; H NMR $7.70(\mathrm{~d}, \mathrm{~J}=8.9,1 \mathrm{H}), 7.66(\mathrm{~d}, \mathrm{~J}=8.9,1 \mathrm{H}), 7.24(\mathrm{~d}, \mathrm{~J}=2.3,1 \mathrm{H}), 7.13(\mathrm{~d}, \mathrm{~J}=8.9$, 1H), $7.03(\mathrm{dd}, \mathrm{J}=8.9,2.3,1 \mathrm{H}), 3.952(\mathrm{~s}, 3 \mathrm{H}), 3.945(\mathrm{~s}, 3 \mathrm{H}), 3.07(\mathrm{t}, \mathrm{J}=7.6,2 \mathrm{H}), 1.74(\mathrm{~m}, 2 \mathrm{H}), 1.69(\mathrm{~m}$, 2H) 1.38 (br s, 1H), $1.23(\mathrm{~s}, 6 \mathrm{H}) ;{ }^{13} \mathrm{C}$ NMR 158.0, 154.8, 134.1, 130.0, 127.1, 124.8, 122.6, 115.5, 110.8, 102.0, 70.9, 56.3, 55.1, 43.8, 29.1, 25.4, 24.4; MS m/e $288\left(\mathrm{M}^{+}\right)$; HRMS m/e for $\mathrm{C}_{18} \mathrm{H}_{24} \mathrm{O}_{3}$ calcd $\left(\mathrm{M}^{+}\right)$288.1725, found 288.1713.

1,6-Dimethoxy-7,7-dimethyl-7,8,9,10-tetrahydrocyclohepta[de]naphthalene $\quad(18) \quad$ and $\quad 2,7-$ Dimethoxy-1-(4-methylpent-3-enyl)naphthalene (19): To a solution of $16(0.0750 \mathrm{~g}, 0.260 \mathrm{mmol})$ in $\mathrm{CH}_{2} \mathrm{Cl}_{2}(10 \mathrm{~mL})$, one drop of $\mathrm{H}_{2} \mathrm{SO}_{4}$ was added. The solution was refluxed for $24 \mathrm{~h}$. Water was added and a conventional extractive workup performed $\mathrm{CH}_{2} \mathrm{Cl}_{2}$ ). Purification by preparative TLC (4:1 hexanes: $\left.\mathrm{CH}_{2} \mathrm{Cl}_{2}\right)$ gave, in order of elution, $\mathbf{1 8}(0.0490 \mathrm{~g}, 70 \%$ yield $)$ and $\mathbf{1 9}(0.0056 \mathrm{~g}, 8 \%$ yield $)$ as a mixture. 18, mp $101{ }^{\circ} \mathrm{C}$. IR $(\mathrm{KBr})_{\max } 2929,1612 ;{ }^{1} \mathrm{H}$ NMR $7.54(\mathrm{~d}, \mathrm{~J}=8.9,1 \mathrm{H}), 7.52(\mathrm{~d}, \mathrm{~J}=8.8,1 \mathrm{H})$, $7.09(\mathrm{~d}, \mathrm{~J}=8.9,1 \mathrm{H}), 7.06(\mathrm{~d}, \mathrm{~J}=8.8,1 \mathrm{H}), 3.94(\mathrm{~s}, 3 \mathrm{H}), 3.91(\mathrm{~s}, 3 \mathrm{H}), 3.00(\mathrm{br} \mathrm{m}, 2 \mathrm{H}), 1.95(\mathrm{~m}, 2 \mathrm{H}), 1.69$ (m, 2H), $1.52(\mathrm{~s}, 6 \mathrm{H}) ;{ }^{13} \mathrm{C}$ NMR 158.8, 154.5, 137.6, 131.7, 127.3, 126.9, 125.4, 122.9, 112.1, 111.6, 57.4, 56.0, 41.1, 39.4, 25.5, 22.1; MS m/e $270\left(\mathrm{M}^{+}\right)$; HRMS m/e for $\mathrm{C}_{18} \mathrm{H}_{22} \mathrm{O}_{2}$ calcd $\left(\mathrm{M}^{+}\right)$270.1620, found 270.1614 .

(19): $55-56{ }^{\circ} \mathrm{C} ;{ }^{1} \mathrm{H}$ NMR $7.69(\mathrm{~d}, \mathrm{~J}=9.4,1 \mathrm{H}), 7.66(\mathrm{~d}, \mathrm{~J}=9.4,1 \mathrm{H}), 7.25(\mathrm{~d}, \mathrm{~J}=2.1,1 \mathrm{H}), 7.13(\mathrm{~d}, \mathrm{~J}=$ 8.7, 1H), $7.02(\mathrm{dd}, \mathrm{J}=8.7,2.1,1 \mathrm{H}), 5.36(7, \mathrm{~J}=7.2,1 \mathrm{~h}), 3.95(\mathrm{~s}, 6 \mathrm{H}), 3.05(\mathrm{~m}, 2 \mathrm{H}), 2.31(\mathrm{~m}, 2 \mathrm{H}), 1.73$ (s, 3H), 1.61 (s, 3H); ${ }^{13} \mathrm{C}$ NMR 158.1, 155.0, 134.2, 131.8, 130.0, 127.2, 124.8, 124.6, 122.7, 115.7, $110.9,102.0,56.5,55.2,28.2,25.7,25.5,17.6$; HRMS m/e for $\mathrm{C}_{18} \mathrm{H}_{22} \mathrm{O}_{2}$ calcd $\left(\mathrm{M}^{+}\right)$270.1620, found 270.1606 


\section{Hexacarbonyl[ $\mu$ - 4-(7-acetoxy-2-methoxy-1-(3-carbomethoxyprop-2-ynyl)naphthalene)]dicobalt}

(21): Subjecting $20(0.249 \mathrm{~g}, 1.152 \mathrm{mmol}), \mathbf{6 b}(0.525 \mathrm{~g}, 1.27 \mathrm{mmol})$ and $\mathrm{BF}_{3}-\mathrm{OEt}_{2}(440 \mu \mathrm{L}, 3.5 \mathrm{mmol})$ to Method 1 afforded 21 (0.609 g, 1.018 mmol, 88\% yield) following flash chromatographic purification (2:1 petroleum ether: $\left.\mathrm{Et}_{2} \mathrm{O}\right)$, as a viscous red-brown oil: $\mathrm{IR}(\mathrm{KBr}){ }_{\max }$ 3004, 2952, 2099, 2063, 2029,1765, 1709; ${ }^{1} \mathrm{H}$ NMR $7.82(\mathrm{~d}, \mathrm{~J}=9.0,1 \mathrm{H}), 7.81(\mathrm{~d}, \mathrm{~J}=8.8,1 \mathrm{H}) 7.62(\mathrm{~d}, \mathrm{~J}=2.2,1 \mathrm{H}), 7.25(\mathrm{~d}, \mathrm{~J}=$ 9.0, 1H), $7.11(\mathrm{dd}, \mathrm{J}=8.8,2.2,1 \mathrm{H}), 4.60(\mathrm{~s}, 2 \mathrm{H}), 3.95(\mathrm{~s}, 3 \mathrm{H}), 3.76(\mathrm{~s}, 3 \mathrm{H}), 2.33(\mathrm{~s}, 3 \mathrm{H}) ;{ }^{13} \mathrm{C} \mathrm{NMR}$ $198.3,170.7,169.6,155.0,149.5,133.4,130.0,129.0,127.0,120.2,118.8,114.3,112.0,98.7,94.1$, 55.4, 52.7, 28.4, 21.0; MS m/e 542( $\left.\mathrm{M}^{+}-2 \mathrm{CO}\right), 514\left(\mathrm{M}^{+}-3 \mathrm{CO}\right), 486\left(\mathrm{M}^{+}-4 \mathrm{CO}\right), 458\left(\mathrm{M}^{+}-5 \mathrm{CO}\right), 430\left(\mathrm{M}^{+}-\right.$ 6CO); HRMS m/e for $\mathrm{C}_{24} \mathrm{H}_{16} \mathrm{Co}_{2} \mathrm{O}_{11}$ calcd ( $\left.\mathrm{M}^{+}-3 \mathrm{CO}\right) 513.9509$, found 513.9511 .

7-Acetoxy-2-methoxy-1-(3-carbomethoxyprop-2-ynyl)naphthalene (22): Subjecting 21 (0.284 g, $0.475 \mathrm{mmol})$ to Method 3, followed by recrystallization from $\mathrm{Et}_{2} \mathrm{O}$ afforded 22 (0.138 $\mathrm{g}, 93 \%$ yield): $\mathrm{mp}$ $132-133{ }^{\circ} \mathrm{C}$; IR (KBr) ${ }_{\max } 2917,2234,1761,1712 ;{ }^{1} \mathrm{H}$ NMR $7.80(\mathrm{~d}, \mathrm{~J}=8.9,1 \mathrm{H}), 7.78(\mathrm{~d}, \mathrm{~J}=8.9$, $1 \mathrm{H}), 7.61(\mathrm{~d}, \mathrm{~J}=1.9,1 \mathrm{H}), 7.23(\mathrm{~d}, \mathrm{~J}=8.9,1 \mathrm{H}), 7.14(\mathrm{dd} . \mathrm{J}=8.9,1.9,1 \mathrm{H}), 4.07(\mathrm{~s}, 2 \mathrm{H}), 3.96(\mathrm{~s}, 3 \mathrm{H})$, 3.70 (s, 3H), 2.38 (s, 3H); ${ }^{13} \mathrm{C}$ NMR 169.4, 154.7, 154.0, 149.5, 133.1, 129.9, 129.2, 126.9, 118.8, 114.8, 113.7, 112.7, 87.4, 72.0, 56.3, 52.3, 21.0, 14.6; MS m/e $312\left(\mathrm{M}^{+}\right)$; HRMS m/e for $\mathrm{C}_{18} \mathrm{H}_{16} \mathrm{O}_{5}$ calcd $\left(\mathrm{M}^{+}\right)$ 312.0998, found 312.0991.

7-Acetoxy-1-(3-carbomethoxypropyl)-2-methoxynaphthalene (23): To a solution of 22 (0.157 g, $0.503 \mathrm{mmol})$ in $\mathrm{MeOH}(15 \mathrm{~mL})$ under $\mathrm{H}_{2}$ was added $\mathrm{Pd} / \mathrm{C}$ (excess). The solution was stirred for $6 \mathrm{~h}$, following which the suspension was filtered and the filtrate concentrated under reduced pressure. Preparative TLC (1:1 petroleum ether: $\left.\mathrm{Et}_{2} \mathrm{O}\right)$ gave $23(0.148 \mathrm{~g}, 93 \%$ yield $)$ as white solid: $\mathrm{mp} 125{ }^{\circ} \mathrm{C}$; IR $(\mathrm{KBr})_{\max } 3067,2950,1759,1734 ;{ }^{1} \mathrm{H}$ NMR $7.79(\mathrm{~d}, \mathrm{~J}=8.8,1 \mathrm{H}), 7.72(\mathrm{~d}, \mathrm{~J}=9.0,1 \mathrm{H}), 7.65(\mathrm{~d}, \mathrm{~J}=$ 2.0, 1H), $7.22(\mathrm{~d}, \mathrm{~J}=9.0,1 \mathrm{H}), 7.11(\mathrm{dd}, \mathrm{J}=8.8,2.0,1 \mathrm{H}), 3.92(\mathrm{~s}, 3 \mathrm{H}), 3.68(\mathrm{~s}, 3 \mathrm{H}), 3.09(\mathrm{t}, \mathrm{J}=7.5,2 \mathrm{H})$, $2.42(\mathrm{t}, \mathrm{J}=7.5,2 \mathrm{H}), 2.37(\mathrm{~s}, 3 \mathrm{H}), 1.98(\mathrm{~m}, 2 \mathrm{H}) ;{ }^{13} \mathrm{C} \mathrm{NMR} 173.9,169.5,154.9,149.1,133.6,129.9$, 127.6, 127.1, 122.3, 118.4, 114.0, 112.7, 56.1, 51.2, 33.5, 24.7, 24.0, 21.1; MS m/e $316\left(\mathrm{M}^{+}\right)$; HRMS $\mathrm{m} / \mathrm{e}$ for $\mathrm{C}_{18} \mathrm{H}_{20} \mathrm{O}_{5}$ calcd $\left(\mathrm{M}^{+}\right)$316.1311, found 316.1316. 
1-(3-Carboxypropyl)-7-hydroxy-2-methoxynaphthalene (24): To a solution of 23 (0.117 g, 0.370

mmol ) in methanol $(20 \mathrm{~mL})$ was added an excess of sodium hydroxide. Following heating to reflux for $18 \mathrm{~h}$, the mixture was acidified $(3 \mathrm{M} \mathrm{HCl})$ and a conventional extractive workup performed $\left(\mathrm{Et}_{2} \mathrm{O}\right)$. Recrystallization from $\mathrm{CH}_{2} \mathrm{Cl}_{2}$ afforded product 24 (0.0730 g, $73 \%$ yield): mp 164-165 ${ }^{\circ} \mathrm{C}$; IR (KBr) ${ }_{\max } 3385$ br, 2924, 1703, 1626; ${ }^{1} \mathrm{H}$ NMR (acetone- $\left.\mathrm{d}_{6}\right) \quad 7.63(\mathrm{~d}, \mathrm{~J}=8.8,1 \mathrm{H}), 7.62(\mathrm{~d}, \mathrm{~J}=9.0,1 \mathrm{H}), 7.28$ (br s,1H), $7.11(\mathrm{~d}, \mathrm{~J}=9.0,1 \mathrm{H}), 6.93(\mathrm{dd}, \mathrm{J}=8.8,2.1,1 \mathrm{H}), 3.87(\mathrm{~s}, 3 \mathrm{H}), 2.99(\mathrm{t}, \mathrm{J}=7.7,2 \mathrm{H}), 2.35(\mathrm{~m}$, 2H), $1.85(\mathrm{~m}, 2 \mathrm{H}) ;{ }^{13} \mathrm{C}$ NMR (acetone-d $)_{6}$ 174.5, 156.0, 155.1, 134.8, 130.2, 127.7, 124.5, 120.7, 115.7, 110.3, 104.8, 55.8, 33.3, 24.8, 24.1; MS m/e $260\left(\mathrm{M}^{+}\right)$; HRMS m/e for $\mathrm{C}_{15} \mathrm{H}_{16} \mathrm{O}_{4}$ calcd $\left(\mathrm{M}^{+}\right)$260.1049, found 260.1045 .

6-Hydroxy-1-methoxy-9,10-dihydrocyclohepta[de]naphthalen-7(8H)-one (25): A solution of polyphosphoric acid (PPA, ca. $0.1 \mathrm{~g}$, excess) and $24(0.100 \mathrm{~g}, 0.385 \mathrm{mmol})$ in $\mathrm{CH}_{2} \mathrm{Cl}_{2}(20 \mathrm{~mL})$, was heated to reflux for $36 \mathrm{~h}$. Water was added and the mixture subjected to a conventional extractive workup $\left(\mathrm{CH}_{2} \mathrm{Cl}_{2}\right)$. Preparative TLC (2:1 petroleum ether: $\left.\mathrm{Et}_{2} \mathrm{O}\right)$ gave $25(0.075 \mathrm{~g}, 80 \%$ yield ); mp 90 ${ }^{\circ} \mathrm{C} ; \mathrm{IR}(\mathrm{KBr})_{\max } 3009,2970,1616 \mathrm{~cm}^{-1} ;{ }^{1} \mathrm{H}$ NMR $12.73(\mathrm{~s}, 1 \mathrm{H}), 7.71(\mathrm{~d}, \mathrm{~J}=8.9,1 \mathrm{H}), 7.63(\mathrm{~d}, \mathrm{~J}=8.8$, $1 \mathrm{H}), 7.12(\mathrm{~d}, \mathrm{~J}=8.8,1 \mathrm{H}), 6.93(\mathrm{~d}, \mathrm{~J}=8.9,1 \mathrm{H}), 3.95(\mathrm{~s}, 3 \mathrm{H}), 3.01(\mathrm{t}, \mathrm{J}=7.1,2 \mathrm{H}), 2.71(\mathrm{t}, \mathrm{J}=7.4,2 \mathrm{H})$, 2.39 (m, 2H); ${ }^{13} \mathrm{C}$ NMR 207.4, 162.5, 157.3, 136.2, 135.7, 128.6, 123.2, 122.4, 116.5, 115.2, 110.3, 56.2, 42.3, 29.5, 25.4; MS m/e $242\left(\mathrm{M}^{+}\right)$; HRMS for $\mathrm{C}_{15} \mathrm{H}_{14} \mathrm{O}_{3}$ calcd $\left(\mathrm{M}^{+}\right)$242.0943, found 242.0931.

7-Hydroxy-1-(4-hydroxy-4-methylpentyl)-2-methoxynaphthalene (26): To a solution of 23 $(0.1030 \mathrm{~g}, 0.3259 \mathrm{mmol})$ in $\mathrm{Et}_{2} \mathrm{O}$ at $0^{\circ} \mathrm{C}$ was added $\mathrm{MeLi}\left(0.76 \mathrm{~mL}, 1.1 \mathrm{mmol}, 1.5 \mathrm{M}\right.$ in $\left.\mathrm{Et}_{2} \mathrm{O}\right)$. After $4 \mathrm{~h}$ of stirring, aqueous $\mathrm{NH}_{4} \mathrm{Cl}$ was added. A conventional workup and preparative TLC (1:1 petroleum ether: $\left.\mathrm{Et}_{2} \mathrm{O}\right)$ gave $26\left(0.0625 \mathrm{~g}, 70 \%\right.$ yield): $\mathrm{mp} 140{ }^{\circ} \mathrm{C}$; IR $(\mathrm{KBr})_{\max } 3358,2967 \mathrm{~cm}^{-1} ;{ }^{1} \mathrm{H}$ NMR 8.10 (br s, $1 \mathrm{H}), 7.65(\mathrm{~d}, \mathrm{~J}=8.8,1 \mathrm{H}), 7.61(\mathrm{~d}, \mathrm{~J}=8.9,1 \mathrm{H}), 7.33(\mathrm{~d}, \mathrm{~J}=2.2,1 \mathrm{H}), 7.08(\mathrm{~d}, \mathrm{~J}=8.9,1 \mathrm{H}), 7.01(\mathrm{dd}, \mathrm{J}=$ 8.8, 2.2, $1 \mathrm{H}), 3.91(\mathrm{~s}, 3 \mathrm{H}), 2.95$ (br t, J = 7.0, 2H), 2.60 (br s, 1H), $1.65(\mathrm{~m}, 4 \mathrm{H}), 1.21(\mathrm{~s}, 6 \mathrm{H}) ;{ }^{13} \mathrm{C} \mathrm{NMR}$ $154.6,154.5,134.4,130.3,127.3,124.5,122.1,115.6,110.7,105.3,72.0,56.4,43.5,29.0,25.4,24.3$; MS m/e $274\left(\mathrm{M}^{+}\right) ; \mathrm{HRMS} \mathrm{m} / \mathrm{e}$ for $\mathrm{C}_{17} \mathrm{H}_{22} \mathrm{O}_{3}$ calcd 274.1569, found 274.1574. 
6-Methoxy-10,10-dimethyl-8,9,10,10a-tetrahydrocyclohepta[de]naphthalen-1(7H)-one (27): To a

solution of $26(0.0625 \mathrm{~g}, 0.228 \mathrm{mmol})$ in $\mathrm{CH}_{2} \mathrm{Cl}_{2}(10 \mathrm{~mL})$, one drop of $\mathrm{H}_{2} \mathrm{SO}_{4}$ was added at $0^{\circ} \mathrm{C}$. After stirring for $0.5 \mathrm{~h}$, a conventional extractive workup $\left(\mathrm{CH}_{2} \mathrm{Cl}_{2}\right)$ followed by preparative TLC (10:1 petroleum ether:Et $\left.{ }_{2} \mathrm{O}\right)$ afforded $27\left(0.0410 \mathrm{~g}, 70 \%\right.$ yield): $\mathrm{mp} 85-87{ }^{\circ} \mathrm{C}$; IR $(\mathrm{KBr})_{\max } 2934,1653,1615$ $\mathrm{cm}^{-1} ;{ }^{1} \mathrm{H}$ NMR $7.29(\mathrm{~d}, \mathrm{~J}=9.7,1 \mathrm{H}), 7.13(\mathrm{~d}, \mathrm{~J}=8.4,1 \mathrm{H}), 6.79(\mathrm{~d}, \mathrm{~J}=8.4,1 \mathrm{H}), 5.97(\mathrm{~d}, \mathrm{~J}=9.7,1 \mathrm{H})$, $3.84(\mathrm{~s}, 3 \mathrm{H}), 3.63(\mathrm{~s}, 1 \mathrm{H}), 3.36(\mathrm{~m}, 1 \mathrm{H}), 2.42(\mathrm{~m}, 1 \mathrm{H}), 1.84(\mathrm{~m}, 1 \mathrm{H}), 1.51(\mathrm{~m}, 1 \mathrm{H}), 1.38(\mathrm{~m}, 1 \mathrm{H}), 1.27$ (m, 1H), 1.17 (s, 3H), $0.66(\mathrm{~s}, 3 \mathrm{H}) ;{ }^{13} \mathrm{C}$ NMR 203.2, 157.7, 145.5, 140.8, 129.4, 127.9, 124.4, 123.5, 108.9, 58.3, 55.7, 43.2, 37.0, 27.5, 24.3, 21.5, 20.2; MS m/e $256\left(\mathrm{M}^{+}\right)$; HRMS m/e for $\mathrm{C}_{17} \mathrm{H}_{20} \mathrm{O}_{2}$ calcd 256.1463 , found 256.1457.

2-Acetoxy-7-benzyloxynaphthalene (28): To a solution of 7-benzyloxy-2-naphthol (2.000 g, 7.991 mmol) in $\mathrm{CH}_{2} \mathrm{Cl}_{2}(50 \mathrm{~mL})$ were added $\mathrm{Et}_{3} \mathrm{~N}(2 \mathrm{~mL}$, excess $)$ and acetic anhydride $(2 \mathrm{~mL}$, excess $)$. After stirring for $1 \mathrm{~h}$, the mixture was subjected to a conventional extractive workup. Recrystallization from $\mathrm{Et}_{2} \mathrm{O}$ afforded 28 (2.1958g, 94\% yield) as a white crystalline solid: mp $121^{\circ} \mathrm{C}$; IR (KBr) ${ }_{\max } 2938,1751$ $\mathrm{cm}^{-1} ;{ }^{1} \mathrm{H}$ NMR $7.78(\mathrm{~d}, \mathrm{~J}=8.9,1 \mathrm{H}), 7.76(\mathrm{~d}, \mathrm{~J}=8.9,1 \mathrm{H}), 7.49(\mathrm{~d}, \mathrm{~J}=7.4,2 \mathrm{H}), 7.45(\mathrm{~d}, \mathrm{~J}=2.2,1 \mathrm{H})$, 7.42 (apparent t, $\mathrm{J}=7.5,2 \mathrm{H}), 7.36(\mathrm{t}, \mathrm{J}=7.5,1 \mathrm{H}), 7.22(\mathrm{dd}, \mathrm{J}=8.8,2.2,1 \mathrm{H}), 7.19(\mathrm{~d}, \mathrm{~J}=2.2,1 \mathrm{H}), 7.10$ $(\mathrm{dd}, \mathrm{J}=9.0,2.2,1 \mathrm{H}), 5.19(\mathrm{~s}, 2 \mathrm{H}), 2.36(\mathrm{~s}, 3 \mathrm{H}) ;{ }^{13} \mathrm{C} 169.5,157.3,149.0,136.7,135.0,129.3,129.1$, 128.6, 128.0, 127.5, 127.0, 118.8, 118.7, 117.5, 107.1, 70.0, 21.1; MS m/e $292\left(\mathrm{M}^{+}\right)$; HRMS for $\mathrm{C}_{19} \mathrm{H}_{16} \mathrm{O}_{3}$ calcd 292.1099, found 292.1097.

\section{Hexacarbonyl[ $\mu$ - 4-(7-acetoxy-2-benzyloxy-1-(3-carbomethoxyprop-2-}

ynyl)naphthalene)]dicobalt (29): To a solution of $28(0.2000 \mathrm{~g}, 0.6849 \mathrm{mmol})$ in $\mathrm{CH}_{2} \mathrm{Cl}_{2}(10 \mathrm{~mL})$ was added $\mathbf{6 b}(0.3120 \mathrm{~g}, 0.7534 \mathrm{mmol})$ and $\mathrm{Bu}_{2} \mathrm{BOTf}(479 \mu \mathrm{L}, 0.479 \mathrm{mmol})$ was added dropwise at $0^{\circ} \mathrm{C}$. After $1 \mathrm{~h}$ of continuous stirring, $\mathrm{NH}_{4} \mathrm{Cl}_{(\mathrm{aq})}$ was added and the mixture was subjected to a conventional extractive workup. The residue was purified by flash chromatography $\left(2: 1\right.$ petroleum ether: $\left.\mathrm{Et}_{2} \mathrm{O}\right)$ to give 29 (0.4150 g, 90\% yield) as a red-brown solid: IR (KBr) ${ }_{\text {max }}$ 2953, 2113, 2063, 2031, 1765, 1708 $\mathrm{cm}^{-1} ;{ }^{1} \mathrm{H}$ NMR $7.80(\mathrm{~d}, \mathrm{~J}=8.8,1 \mathrm{H}), 7.78(\mathrm{~d}, \mathrm{~J}=9.0,1 \mathrm{H}), 7.65(\mathrm{~d}, \mathrm{~J}=2.1,1 \mathrm{H}), 7.46(\mathrm{~d}, \mathrm{~J}=7.2,2 \mathrm{H})$, 7.39 (apparent t, $\mathrm{J}=7.2,2 \mathrm{H}), 7.33(\mathrm{t}, \mathrm{J}=7.2,1 \mathrm{H}), 7.31(\mathrm{~d}, \mathrm{~J}=9.0,1 \mathrm{H}), 7.14(\mathrm{dd}, \mathrm{J}=8.8,2.1,1 \mathrm{H}), 5.28$ 
(s, 2H), 4.68 (br s, 2H), 3.64 (s, 3H), 2.34 (s, 3H); ${ }^{13} \mathrm{C} 198.2,170.5,169.5,154.2,149.5,136.8,133.5$, $130.1,128.9,128.6,128.0,127.34,127.25,121.0,119.0,114.6,113.9,98.3,78.8,70.9,52.6,28.9,21.0$; MS m/e $618\left(\mathrm{M}^{+}-2 \mathrm{CO}\right), 590\left(\mathrm{M}^{+}-3 \mathrm{CO}\right), 506\left(\mathrm{M}^{+}-6 \mathrm{CO}\right)$; HRMS m/e for $\mathrm{C}_{30} \mathrm{H}_{20} \mathrm{Co}_{2} \mathrm{O}_{11}$ calcd $\left(\mathrm{M}^{+}-2 \mathrm{CO}\right)$ 617.9771, found 617.9773.

\section{7-Acetoxy-2-benzyloxy-1-(3-carbomethoxyprop-2-ynyl)naphthalene (30): Subjecting 29 (0.3630}

g, $0.5385 \mathrm{mmol}$ ) to Method 3 gave the crude reaction product, which upon recrystallization from methanol gave $30\left(0.1880 \mathrm{~g}, 90 \%\right.$ yield): $\mathrm{mp} 144{ }^{\circ} \mathrm{C}$; IR $(\mathrm{KBr}){ }_{\max } 2956,2234,1761,1712 \mathrm{~cm}^{-1} ;{ }^{1} \mathrm{H}$ NMR $7.79(\mathrm{~d}, \mathrm{~J}=8.8,1 \mathrm{H}), 7.75(\mathrm{~d}, \mathrm{~J}=9.0,1 \mathrm{H}), 7.67(\mathrm{~d}, \mathrm{~J}=1.5,1 \mathrm{H}), 7.49(\mathrm{~d}, \mathrm{~J}=7.5,2 \mathrm{H}), 7.43$ (apparent $\mathrm{t}, \mathrm{J}=7.5,2 \mathrm{H}), 7.36(\mathrm{t}, \mathrm{J}=7.5,1 \mathrm{H}), 7.26(\mathrm{~d}, \mathrm{~J}=9.0,1 \mathrm{H}), 7.17(\mathrm{dd}, \mathrm{J}=8.8,1.5,1 \mathrm{H}), 5.25(\mathrm{~s}$, $2 \mathrm{H}), 4.13(\mathrm{~s}, 2 \mathrm{H}), 3.72(\mathrm{~s}, 3 \mathrm{H}), 2.39(\mathrm{~s}, 3 \mathrm{H}) ;{ }^{13} \mathrm{C} 169.5,154.14,154.09,149.6,136.8,133.3,130.1$, $129.3,128.6,128.1,127.3,119.2,116.0,114.4,114.1,87.4,72.3,71.4,52.4,21.2,15.1$; MS m/e 388 $\left(\mathrm{M}^{+}\right)$; HRMS m/e for $\mathrm{C}_{24} \mathrm{H}_{20} \mathrm{O}_{5}$ calcd. 388.1311, found 388.1298.

7-Acetoxy-2-benzyloxy-1-(3-carbomethoxypropyl)naphthalene (31): To a solution of 30 (0.2000 g, $0.5154 \mathrm{mmol}$ ) in ethyl acetate $(20 \mathrm{~mL})$ under $\mathrm{H}_{2}$ was added $\mathrm{Rh} / \mathrm{C}$ (excess) at room temperature. The solution was stirred for $18 \mathrm{~h}$ with monitoring by TLC. The suspension was filtered and the solvent was removed under reduced pressure. Preparative TLC (2:1 petroleum ether: $\left.\mathrm{Et}_{2} \mathrm{O}\right)$ to give $31(0.1880 \mathrm{~g}$, 93\% yield): bp $180-185{ }^{\circ} \mathrm{C}\left(0.15\right.$ torr); IR (KBr) ${ }_{\max } 2956,1763,1731 \mathrm{~cm}^{-1} ;{ }^{1} \mathrm{H}$ NMR $7.81(\mathrm{~d}, \mathrm{~J}=8.8$, 1H), $7.73(\mathrm{~d}, \mathrm{~J}=2.1,1 \mathrm{H}), 7.72(\mathrm{~d}, \mathrm{~J}=8.9,1 \mathrm{H}), 7.50(\mathrm{~d}, \mathrm{~J}=7.4,2 \mathrm{H}), 7.44$ (apparent $\mathrm{t}, \mathrm{J}=7.4,2 \mathrm{H}), 7.37$ $(\mathrm{t}, \mathrm{J}=7.4,1 \mathrm{H}), 7.29(\mathrm{~d}, \mathrm{~J}=8.9,1 \mathrm{H}), 7.16(\mathrm{dd}, \mathrm{J}=8.8,2.1,1 \mathrm{H}), 5.22(\mathrm{~s}, 2 \mathrm{H}), 3.64(\mathrm{~s}, 3 \mathrm{H}), 3.20(\mathrm{t}, \mathrm{J}=$ 7.7, 2H), $2.46(\mathrm{t}, \mathrm{J}=7.4,2 \mathrm{H}), 2.40(\mathrm{~s}, 3 \mathrm{H}), 2.05(\mathrm{~m}, 2 \mathrm{H}) ;{ }^{13} \mathrm{C} 173.8,169.5,154.0,149.0,137.2,133.6$, $129.8,128.4,127.7,127.5,127.3,127.0,123.0,118.6,114.2,114.1,70.9,51.2,33.6,24.7,24.3,21.0$; MS m/e $392\left(\mathrm{M}^{+}\right)$; HRMS m/e for $\mathrm{C}_{24} \mathrm{H}_{24} \mathrm{O}_{5}$ calcd. 392.1624, found 392.1610.

7-Acetoxy-1-(3-carbomethoxypropyl)-2-hydroxynaphthalene (32): To a solution of 31 (0.1900 g, $0.4846 \mathrm{mmol}$ ) in ethyl acetate $(20 \mathrm{~mL})$ under $\mathrm{H}_{2}, \mathrm{Pd} / \mathrm{C}$ (excess) at room temperature. The solution was stirred for $4 \mathrm{~h}$ with monitoring by TLC. The suspension was filtered and the solvent was removed under 
reduced pressure. Preparative TLC (1:1 petroleum ether: $\left.\mathrm{Et}_{2} \mathrm{O}\right)$ afforded $32(0.1400 \mathrm{~g}, 96 \%$ yield), mp 80-81 ${ }^{\circ} \mathrm{C}: \mathrm{IR}(\mathrm{KBr}) \quad \max 3425 \mathrm{br}, 2952,1759,1733 \mathrm{~cm}^{-1}$; ${ }^{1} \mathrm{H}$ NMR $7.75(\mathrm{~d}, \mathrm{~J}=8.8,1 \mathrm{H}), 7.572(\mathrm{~d}, \mathrm{~J}=$ 8.8, 1H), $7.565(\mathrm{~d}, \mathrm{~J}=2.3,1 \mathrm{H}), 7.25(\mathrm{br}, 1 \mathrm{H}), 7.073(\mathrm{~d}, \mathrm{~J}=8.8,1 \mathrm{H}), 7.069(\mathrm{dd}, \mathrm{J}=8.8,2.3,1 \mathrm{H}), 3.74$ (s, 3H), $3.02(\mathrm{t}, \mathrm{J}=7.8,2 \mathrm{H}), 2.43(\mathrm{t}, \mathrm{J}=6.8,2 \mathrm{H}), 2.38(\mathrm{~s}, 3 \mathrm{H}), 1.96(\mathrm{~m}, 2 \mathrm{H}) ;{ }^{13} \mathrm{C} 175.6,170.0,152.5$, 149.0, 133.7, 130.0, 127.7, 127.1, 118.3, 118.2, 117.7, 113.7, 51.9, 32.6, 24.3, 23.8, 21.2; Ms m/e 302 $\left(\mathrm{M}^{+}\right)$; HRMS m/e for $\mathrm{C}_{17} \mathrm{H}_{18} \mathrm{O}_{5}$ calcd. 302.1154, found 302.1141.

\section{7-Acetoxy-1-(3-carbomethoxypropyl)-2-(trifluoromethylsulfonyloxy)naphthalene $\quad(33)$ : $\quad$ To a}

solution of $32(0.1700 \mathrm{~g}, 0.5629 \mathrm{mmol})$ in $\mathrm{CH}_{2} \mathrm{Cl}_{2}(20 \mathrm{~mL})$, pyridine $(136 \mu \mathrm{L}, 1.69 \mathrm{mmol})$ and $\mathrm{Tf}_{2} \mathrm{O}(105$ $\mu \mathrm{L}, 0.625 \mathrm{mmol}$ ), were added sequentially. The reaction mixture was stirred for $0.5 \mathrm{~h}$. Following a coventional extractive workup $\left(\mathrm{CH}_{2} \mathrm{Cl}_{2}\right)$, preparative TLC (1:1 petroleum ether: $\left.\mathrm{Et}_{2} \mathrm{O}\right)$ afforded 33 $(0.2347 \mathrm{~g}, 96 \%$ yield $)$ as a viscous oil: $\mathrm{IR}(\mathrm{KBr}){ }_{\max } 2955,1765,1738 \mathrm{~cm}^{-1} ;{ }^{1} \mathrm{H}$ NMR $7.91(\mathrm{~d}, \mathrm{~J}=8.9$, $1 \mathrm{H}), 7.86(\mathrm{~d}, \mathrm{~J}=2.1,1 \mathrm{H}), 7.80(\mathrm{~d}, \mathrm{~J}=9.1,1 \mathrm{H}), 7.37(\mathrm{~d}, \mathrm{~J}=9.1,1 \mathrm{H}), 7.35(\mathrm{dd}, \mathrm{J}=8.9,2.1,1 \mathrm{H}), 3.72(\mathrm{~s}$, $3 \mathrm{H}), 3.17(\mathrm{~m}, 2 \mathrm{H}), 2.48(\mathrm{t}, \mathrm{J}=7.3,2 \mathrm{H}), 2.40(\mathrm{~s}, 3 \mathrm{H}), 2.03(\mathrm{~m}, 2 \mathrm{H}) ;{ }^{13} \mathrm{C} 173.4,169.4,149.8,145.5$, $133.2,130.7,130.3,129.8,128.8,122.2,119.1,118.5\left(\mathrm{q}, \mathrm{J}_{\mathrm{CF}}=319.8\right), 115.9,51.6,33.4,25.6,24.8$, 21.1; MS m/e $434\left(\mathrm{M}^{+}\right)$; HRMS m/e for $\mathrm{C}_{18} \mathrm{H}_{17} \mathrm{~F}_{3} \mathrm{O}_{7} \mathrm{~S}$ calcd. 434.0647, found 434.0641.

\section{7-Acetoxy-1-(3-carbomethoxypropyl)-2-methylnaphthalene (34) and 1-(3-Carbomethoxypropyl)-}

7-hydroxy-2-methylnaphthalene (35): To a solution of $\mathrm{Pd}_{2}(\mathrm{dba})_{3}(0.0058 \mathrm{~g}, 0.0063 \mathrm{mmol})$ and (2biphenyl)dicyclohexylphosphine $(0.0045 \mathrm{~g}, 0.013 \mathrm{mmol})$ in anhydrous THF $(20 \mathrm{~mL}), \mathrm{DABAL}^{-\mathrm{Me}_{3}}$ (0.0987 $\mathrm{g}, 0.385 \mathrm{mmol}$ in THF) and $33(0.1860 \mathrm{~g}, 0.4281 \mathrm{mmol}$, in THF $(2 \mathrm{~mL}))$ were added sequentially. After $0.5 \mathrm{~h}$ of stirring, dilute $\mathrm{HCl}(1 \mathrm{M})$ was added. After conventional extractive workup $\left(\mathrm{Et}_{2} \mathrm{O}\right)$, the residue was subjected to preparative TLC, which afforded, in order of elution (1:1 petroleum ether: $\left.\mathrm{Et}_{2} \mathrm{O}\right), 34\left(0.1080 \mathrm{~g}, 84 \%\right.$ yield), and $\mathbf{3 5}\left(0.0080 \mathrm{~g}, 7 \%\right.$ yield); (34) $\mathrm{mp} 48{ }^{\circ} \mathrm{C} ;(\mathrm{KBr}){ }_{\max } 2951$, $1769,1736 \mathrm{~cm}^{-1} ;{ }^{1} \mathrm{H}$ NMR $7.81(\mathrm{~d}, \mathrm{~J}=9.0,1 \mathrm{H}), 7.73(\mathrm{~d}, \mathrm{~J}=2.0,1 \mathrm{H}), 7.63(\mathrm{~d}, \mathrm{~J}=8.5,1 \mathrm{H}), 7.29(\mathrm{~d}, \mathrm{~J}$ $=8.5,1 \mathrm{H}), 7.19(\mathrm{dd}, \mathrm{J}=9.0,2.0,1 \mathrm{H}), 3.72(\mathrm{~s}, 3 \mathrm{H}), 3.06(\mathrm{~m}, 2 \mathrm{H}), 2.51(\mathrm{~s}, 3 \mathrm{H}), 2.50(\mathrm{t}, \mathrm{J}=7.0,2 \mathrm{H}), 2.39$ (s, 3H), $1.96(\mathrm{~m}, 2 \mathrm{H}) ;{ }^{13} \mathrm{C} 173.8,169.8,148.6,134.5,133.9,132.6,130.6,129.9,129.0,126.1,119.7$, 
114.6, 51.5, 33.9, 28.0, 24.8, 21.2, 20.1; MS m/e $300\left(\mathrm{M}^{+}\right)$; HRMS m/e for $\mathrm{C}_{18} \mathrm{H}_{20} \mathrm{O}_{4}$ calcd. 300.1362, found 300.1351.

(35): (KBr) ${ }_{\max } 3395$ br. 2953, $1736 \mathrm{~cm}^{-1} ;{ }^{1} \mathrm{H}$ NMR $7.70(\mathrm{~d}, \mathrm{~J}=9.0,1 \mathrm{H}), 7.56(\mathrm{~d}, \mathrm{~J}=8.5,1 \mathrm{H}), 7.44$ (d, $\mathrm{J}=2.3,1 \mathrm{H}), 7.14(\mathrm{~d}, \mathrm{~J}=9.0,1 \mathrm{H}), 7.08(\mathrm{dd}, \mathrm{J}=8.5,2.3,1 \mathrm{H}), 3.76(\mathrm{~s}, 3 \mathrm{H}), 3.01(\mathrm{~m}, 2 \mathrm{H}), 2.52(\mathrm{t}, \mathrm{J}=7.0$, 2H), 2.47 (s, 3H), $1.95(\mathrm{~m}, 2 \mathrm{H}) ;{ }^{13} \mathrm{C} 174.4,153.9,133.6,133.5,133.1,130.4,127.9,126.8,126.1,116.4$, 105.9, 51.7, 33.9, 28.2, 24.5, 20.1; MS m/e $258\left(\mathrm{M}^{+}\right)$; HRMS m/e for $\mathrm{C}_{16} \mathrm{H}_{18} \mathrm{O}_{3}$ calcd. 258.1256, found 258.1259.

7-Hydroxy-1-(4-hydroxy-4-methylpentyl)-2-methylnaphthalene (36): To a solution of 34 (0.0380 $\mathrm{g}, 0.127 \mathrm{mmol})$ in $\mathrm{Et}_{2} \mathrm{O}$ at $0^{\circ} \mathrm{C}$, MeLi $\left(0.59 \mathrm{~mL}, 1.5 \mathrm{M}\right.$ in $\left.\mathrm{Et}_{2} \mathrm{O}, 0.89 \mathrm{mmol}\right)$ was added. After $4 \mathrm{~h}$ of stirring, aqueous $\mathrm{NH}_{4} \mathrm{Cl}$ was added. After a conventional extractive workup $\left(\mathrm{Et}_{2} \mathrm{O}\right)$, preparative TLC (1:1 petroleum ether: $\left.\mathrm{Et}_{2} \mathrm{O}\right)$ gave product $36\left(0.0306 \mathrm{~g}, 94 \%\right.$ yield): $\mathrm{mp} 145-146{ }^{\circ} \mathrm{C}$; IR (KBr) ${ }_{\max } 3312$ (br), 2968, $1635 \mathrm{~cm}^{-1} ;{ }^{1} \mathrm{H}$ NMR $\quad\left(\mathrm{DMSO}_{-}\right) 9.57(\mathrm{~s}, 1 \mathrm{H}), 7.67(\mathrm{~d}, \mathrm{~J}=8.8,1 \mathrm{H}), 7.51(\mathrm{~d}, \mathrm{~J}=8.3,1 \mathrm{H})$, $7.25(\mathrm{~d}, \mathrm{~J}=2.1,1 \mathrm{H}), 7.07(\mathrm{~d}, \mathrm{~J}=8.3,1 \mathrm{H}), 6.99(\mathrm{dd}, \mathrm{J}=8.8,2.1,1 \mathrm{H}), 4.09(\mathrm{~s}, 1 \mathrm{H}), 2.87(\mathrm{~m}, 2 \mathrm{H}), 2.41$ (s, 3H), 1.57 (m, 4H), 1.08 (s, 6H); ${ }^{13} \mathrm{C}$ (DMSO) 155.4, 133.7, 133.3, 132.4, 129.8, 126.8, 125.8, 125.5, 116.9, 105.1, 68.7, 44.0, 29.3, 28.8, 24.3, 19.8; MS m/e $258\left(\mathrm{M}^{+}\right)$; HRMS for $\mathrm{C}_{17} \mathrm{H}_{22} \mathrm{O}_{2}$ calcd. $\left(\mathrm{M}^{+}\right)$ 258.1620, found 258.1620.

\section{6,10,10-Trimethyl-8,9,10,10a-tetrahydrocyclohepta[de]naphthalen-1(7H)-one (37): To a solution} of $36(0.0260 \mathrm{~g}, 0.101 \mathrm{mmol})$ in $\mathrm{CH}_{2} \mathrm{Cl}_{2}(10 \mathrm{~mL}), \mathrm{H}_{2} \mathrm{SO}_{4}(1$ drop $)$ was added and the solution was stirred for $4 \mathrm{~h}$. A conventional extractive workup $\left(\mathrm{CH}_{2} \mathrm{Cl}_{2}\right)$ followed by preparative TLC (1:4 hexanes: $\left.\mathrm{CH}_{2} \mathrm{Cl}_{2}\right)$ afforded product $37\left(0.0210 \mathrm{~g}, 87 \%\right.$ yield): $\mathrm{mp} 73-74{ }^{\circ} \mathrm{C}$; IR (KBr) ${ }_{\text {max }} 2955,1654 \mathrm{~cm}^{-1} ;{ }^{1} \mathrm{H}$ NMR 7.33 $(\mathrm{d}, \mathrm{J}=9.8,1 \mathrm{H}), 7.11(1 / 2 \mathrm{AB}$ quartet, $\mathrm{J}=7.7,1 \mathrm{H}), 7.08(1 / 2$ Abquartet, $\mathrm{J}=7.7,1 \mathrm{H}), 6.05(\mathrm{~d}, \mathrm{~J}=9.8$, 1H), $3.66(\mathrm{~s}, 1 \mathrm{H}), 2.99(\mathrm{~m}, 1 \mathrm{H}), 2.70(\mathrm{~m}, 1 \mathrm{H}), 2.36(\mathrm{~s}, 3 \mathrm{H}), 1.90(\mathrm{~m}, 1 \mathrm{H}), 1.55(\mathrm{~m}, 1 \mathrm{H}), 1.32(\mathrm{~m}, 1 \mathrm{H})$, $1.21(\mathrm{~s}, 3 \mathrm{H}), 1.19(\mathrm{~m}, 1 \mathrm{H}), 0.67(\mathrm{~s}, 3 \mathrm{H}) ;{ }^{13} \mathrm{C} 203.6,145.7,138.9,136.9,128.9,128.1,126.6,125.9,58.2$, 42.3, 37.4, 29.7, 26.8, 25.6, 24.9, 20.4, 19.9; MS m/e $240\left(\mathrm{M}^{+}\right)$; HRMS m/e for $\mathrm{C}_{17} \mathrm{H}_{20} \mathrm{O}$ calcd. 240.1514, found 240.1518 . 
tetrahydrocyclohepta[de]naphthalen-1(7H)-one 2,3-oxide (39): To a solution of 37 (0.0200 g, 0.0833 mmol) in dry DMF $(3 \mathrm{~mL}), \mathrm{NaH}(0.0024 \mathrm{~g}, 0.10 \mathrm{mmol})$ was added. After stirring of reaction mixture for $3 \mathrm{~h}$ in open air, the reaction was subjected to a conventional extractive workup $\left(\mathrm{Et}_{2} \mathrm{O}\right)$. Prepartive TLC (1:1 hexanes : $\left.\mathrm{CH}_{2} \mathrm{Cl}_{2}\right)$ gave, in order of elution, 38 (0.0090 g, $42 \%$ yield), and $\mathbf{3 9}(0.0070 \mathrm{~g}, 31 \%$ yield $)$; (38): $\mathrm{mp} 102{ }^{\circ} \mathrm{C}$; IR (KBr) ${ }_{\max } 3450,2959,1657 \mathrm{~cm}^{-1} ;{ }^{1} \mathrm{H}$ NMR $7.29(\mathrm{~d}, \mathrm{~J}=9.8,1 \mathrm{H}), 7.10(\mathrm{~d}, \mathrm{~J}=7.6$, 1H), $6.95(\mathrm{~d}, \mathrm{~J}=7.6,1 \mathrm{H}), 6.20(\mathrm{~d}, \mathrm{~J}=9.8,1 \mathrm{H}), 4.40(\mathrm{~s}, 1 \mathrm{H}), 3.64(\mathrm{~m}, 1 \mathrm{H}), 2.79(\mathrm{~m}, 1 \mathrm{H}), 2.34(\mathrm{~s}, 3 \mathrm{H})$, $2.33(\mathrm{~m}, 1 \mathrm{H}), 1.83(\mathrm{~m}, 1 \mathrm{H}), 1.45(\mathrm{~m}, 1 \mathrm{H}), 1.29(\mathrm{~m}, 1 \mathrm{H}), 0.82(\mathrm{~s}, 6 \mathrm{H}) ;{ }^{13} \mathrm{C} 205.6,148.0,143.5,140.4$, $138.8,130.2,128.8,127.3,123.1,84.4,42.4,39.0,27.9,26.8,23.2,22.0,21.5 ; \mathrm{MS} \mathrm{m} / \mathrm{e} 256\left(\mathrm{M}^{+}\right)$; HRMS m/e for $\mathrm{C}_{17} \mathrm{H}_{20} \mathrm{O}_{2}$ calcd. 256.1463, found 256.1473.

(39): $\mathrm{mp} 110-11{ }^{\circ} \mathrm{C} \mathrm{IR}(\mathrm{KBr})_{\max } 3456,2924,1698 \mathrm{~cm}^{-1} ;{ }^{1} \mathrm{H}$ NMR 7.15 (1/2 AB quartet, J = 7.7, 1H), $7.11(1 / 2$ AB quartet, $\mathrm{J}=7.7,1 \mathrm{H}), 4.37(\mathrm{~s}, 1 \mathrm{H}), 4.21(\mathrm{~d}, \mathrm{~J}=4.2,1 \mathrm{H}), 3.95(\mathrm{~d}, \mathrm{~J}=4.2,1 \mathrm{H}), 3.46$ $(\mathrm{dd}, \mathrm{J}=14.3,14.3,1 \mathrm{H}), 2.87(\mathrm{dd}, \mathrm{J}=14.3,5.9,1 \mathrm{H}), 2.49(\mathrm{~m}, 1 \mathrm{H}), 2.35(\mathrm{~s}, 3 \mathrm{H}), 1.78(\mathrm{~m}, 1 \mathrm{H}), 1.58(\mathrm{~m}$, 1H), $1.26(\mathrm{~m}, 1 \mathrm{H}), 1.03(\mathrm{~s}, 3 \mathrm{H}), 0.84(\mathrm{~s}, 3 \mathrm{H}) ;{ }^{13} \mathrm{C} 212.1,143.9,139.3,138.3,130.2,127.5,127.2,85.2$, 59.5, 54.4, 43.7, 39.5 , 28.7, 27.8, 25.7, 23.4, 21.7; MS m/e $272\left(\mathrm{M}^{+}\right)$; HRMS m/e for $\mathrm{C}_{17} \mathrm{H}_{20} \mathrm{O}_{3}$ calcd. 272.1412, found 272.1404. ${ }^{1} \mathrm{H}$ NOESY spectral studies showing cross peaks between the $0.84 \mathrm{ppm}$ (methyl) and 4.37 ppm (hydroxy) resonances, along with cross peaks between 1.03 ppm (methyl) and both the $3.95 \mathrm{ppm}$ and $4.21 \mathrm{ppm}$ (epoxide $\mathrm{CH}$ ) resonances support a cis- relationship between the $\mathrm{OH}$ and epoxy functions.

\section{References}

(1) (a) Ulubelen, A.; Topçu, G.; Tan, N.; Lin, L.-J.; Cordell, G. A. Phytochemistry 1992, 31, 24192421; (b) Ulubelen, A.; Topçu, G.; Tan, N. Phytochemistry 1992, 31, 3637-3638; (c) Topçu, G.; Ulubelen, A. J. Nat. Prod. 1996, 59, $734-737$ (d) Ulubelen, A.; Topçu, G.; Ufuk Sönmez, U.; M. Iqbal Choudhary, M.; Atta-Ur-Rahman Phytochemistry 1995, 40, 861-864; (e) Ulubelen, A.; Sönmez, U.; 
Topçu, G. Phytochemistry 1997, 44, 1297-1299; (f) Ulubelen, A.; Tan, N.; Topçu, G. Phytochemistry 1997, 45, 1221-1223; (g) Sönmez, U.; Topçu, G.; Ulubelen, A. Turk. J. Chem. 1997, 21, 376-382; (h) Ulubelen, A.; Topçu; G.; Chai, H.-B.; Pezzuto, J. M. Pharm. Biol. 1999, 37, 148-151; (i) Li, M.; Zhang, J.-S.; Ye, Y.-M.; Fang, J.-N. J. Nat. Prod. 2000, 63, 139-141; (j) Aboul-Ela, M. A.; El-Lakany, A. M. Alexandria J. Pharm. Sci. 2000, 14, 57-61; (k) Ulubelen, A.; Oksuz, S.; Kolak, U.; Bozok-Johansson, C.; Celik, C.; Voelter, W. Planta Med. 2000, 66, 458-462; (1) Okodil, G.; Topçu, G.; Goren, A. C.; Voelter, W. Z. Naurfoscht. B 2002, 57, 957-960; (m) Topçu, G.; Altiner, E. N.; Gozcu, S.; Halfon, B.; Aydogmus, Z.; Pezzuto, J. M.; Zhou, B.-N.; Kingston, D.G. I. Planta Med. 2003, 69, 462-464; (n) A. Kabouche, A.; N. Boutaghane, N.; Z. Kabouche, Z.; E. Seguin, E.; F. Tillequin, F.; K. Benlabed, K. Filoterapia 2005, 76, 450-452.

(2) Chyu, C.-F.; Lin, H.-C; Kuo, Y.-H. Chem. Pharm. Bull. 2005, 53, 11-14.

(3) (a) Topçu, G.; Gören, A. C. Rec. Nat. Prod. 2007, 1, 1-16; (b) Ulubelen, A. In Sage. The Genus Salvia; Kintzios, S. E., Ed.; Harwood: Amsterdam, 2000; pp. 55-68.

(4) (a) Fieser, L. F.; Peters, M. A. J. Am. Chem. Soc. 1932, 54, 4347-4356; (b) Morita, K.; Aida, T.; Morinaga, K.; Tsunetsugu, J. J. Chem. Soc., Perkin Trans. 1 1994, 1215-1220; (c) Ashworth, G.; Barry, D.; Smith, D. C. C. J. Chem. Soc., Perkin Trans. 1 1979, 2995-3000.

(5) (a) Gleiter, R.; Dobler, W. Chem. Ber. 1985, 118, 4725-4742.; (b) Tsunetsugu, J.; Yamaguchi, T.; Ebina, S.; Morinaga, K. J. Chem. Soc, Perkin Trans 1 1986, 1965-1973; (c) Sheilds, J. E.; Gavrilovoc, D.; Kopecký, J.; Hartmann, W.; Heine, H.-G. J. Org. Chem. 1974, 39, 515-520.

(6) Boekelheide, V.; Vick, G. K. J. Am. Chem. Soc. 1956, 78, 653-658.

(7) Vogel, E.; Neumann, B.; Klug, W.; Schmickler, H.; Lex, J. Angew. Chem.Int. Ed. Engl. 1985, 24, 1046-1048; Angew.Chem. 1985, 97, 1044-1045. 
(8) Connolly, T. J.; Durst, T. Tetrahedron 1997, 53, 15969-15982.

(9) In addition, some homologs, such as the hypocrellins, have seen recent synthetic attaention; (a) O'Brien, E.M.; Morgan, B.J.; Mulrooney, C.A.; Carroll, P.J.; Kozlowski, M.C. J. Org. Chm. 2010, 75, 57-68; (b) O'Brien, E.M.; Li, J. Carroll, P.J.; Kozlowski, M.C. J. Org. Chem. 2010, 75, 69-73.

(10) Green, J. R. Eur. J. Org. Chem. 2008, 6053-6062.

(11) For recent reviews dedicated to the Nicholas reaction: (a) Diaz, D. D.; Betancort, J. M.; Martin, V. S. Synlett 2007, 343-353; (b) Teobald, B. J. Tetrahedron 2002, 58, 4133-4170; (c) Green, J. R. Curr. Org. Chem. 2001, 5, 809-826. For reviews covering Nicholas reactions in part, see reference 9 and: (d) Fletcher, A. J.; Christie, S. D. R. J. Chem. Soc., Perkin Trans 1 2000, 1657-1668; (e) Welker, M. E. Curr. Org. Chem. 2001, 5, 785-807; (f) Müller, T. J. J. Eur. J. Org. Chem. 2001, 2021-2033; (g) Omae, I. Appl. Organometal. Chem. 2007, 21, 318-344; (h) Bromfield, K. M.; Gradén, H.; Ljungdahl, N.; Kann, N. Dalton Trans. 2009, 5051-5061.

(12) (a) Green, J. R.; Tjeng, A. A. J. Org. Chem. 2009, 74, 7411-7416; (b) Green, J. R. Chem. Commun. 1998, 1751-1752; (d) Vizniowski, C. S.; Green, J. R.; Breen, T. L.; Dalacu, A. V. J. Org. Chem. 1995, 60, 7496-7502.

(13) For -carbonyl cation equivalents by way of cationic allyl iron complexes, see: a) Green, J.R.; Carroll, M.K. Tetrahedron Lett. 1991, 32, 1141-1144; b) Zhou, T.; Green, J. R. Tetrahedron Lett. 1993, 34, 4497-4500; c) Charlton, M. A., Green, J. R. Can. J. Chem. 1997, 75, 965-974; d) Enders, D.; Jandeleit, B.; Von Berg, S. Synlett 1997, 421-431, and references therein.

(14) For reviews including -carbonyl cation equivalents and their vinylogous homologs by way of iron dienyl cations, see: a) Pearson, A. J. Adv. Met.-Org. Chem. 1988, 1, 1-49, and references therein; b) Pearson, A. J. Iron Compounds in Organic Synthesis; Academic: London, 1994; Chapter 5; (c) 
Donaldson, W. A.; Chaudhury, S. Eur. J. Org. Chem. 2009, 3831-3843; (d) Donaldson, W. A. Curr. Org. Chem. 2000, 4, 837-868.

(15) For -carbonyl cation equivalents by of carbonyl substituted cyclopropanes, see: a) Lifchits, O.; Alberico, D.; Zakharian, I.; Charette, A. B. J. Org. Chem. 2008, 73, 6838, and references therein; b) Hu, B.; Xing, S.; Wang, Z. Org. Lett. 2008, 10, 5481-5484; c) Harrington, P. A.; Kerr, M. A. Tetrahedron Lett. 1997, 38, 5949; d) Danishefsky, S. Acc. Chem. Res. 1979, 12, 66.

(16) For a preliminary report of this work: Taj, R.; Green, J. R. Synlett 2009, 292-296.

(17) Richer, J.-C.; Pépin, Y. Can. J. Chem. 1965, 43, 3443-3445.

(18) Shorthill, B. J.; Glass, T. E. Org. Lett. 2001, 3, 577-579.

(19) (a) Kotha, S.; Mandal, K.; Tiwari, A.; Mobin, S. M. Chem. Eur. J. 2006, 12, 8024-8038. (b) Chattopadhyay, S. K.; Ghosh, D.; Neogi, K. Synth. Commun. 2007, 37, 1535-1543.

(20) (a) Gorelik, A. M.; Reznichenko, A. V.; Andronova, N. A.; Luk'yanets, E. A. J. Org. Chem. USSR 1983, 19, 183-189; (b) Bell, K. H.; McCaffery, L. F. Aust. J. Chem. 1992, 45, 1213-1224.

(21) The superiority of $\mathrm{Bu}_{2} \mathrm{BOTf}$ over $\mathrm{BF}_{3}-\mathrm{OEt}_{2}$ in Nicholas reactions for substrates possessing Lewis basic groups has been observed in our group previously; (a) Green, J. R. Chem. Commun., 1998, 17511752; (b) Green, J. R.; Tjeng, A. A. J. Org. Chem. 2009, 74, 7411-7416.

(22) a) Rieche, A.; Fruhwald, E. Chem. Ber. 1931, 64B, 1603-1606.; b) Moghaddam, F. M.; Porkaleh, H.; Zali-Boeini, H. Lett. Org. Chem. 2006, 3, 123-127..

(23) (a) Biswas, K.; Prieto, O.; Goldsmith, P. J.; Woodward, S. Angew. Chem. Int. Ed. 2005, 44, $2232-$ 2234; Angew. Chem. 2005, 117, 2272-2274; (b) Cooper, T.; Novak, A.; Humphreys, L. D.; Walker, M. D.; Woodward, S. Adv. Synth. Catal. 2006, 348. 686-690. 
(24) Ishikawa, T.; Hino, K.; Yoneda, T.; Murota, M.; Yamaguchi, K.; Watanabe, T. J. Org. Chem. 1999, 64, 5691-5695.

(25) Haraldsson, Gudmundur G.; Baldwin, Jack E. Tetrahedron 1997, 53, 215-224.

(26) D'Agostino, M. F.; Frampton, C. S.; McGlinchey, M. J. Organometallics 1990, 9, 2972-2984.

(27) Tjeng, A. A.; Green, J. R. J. Org. Chem. 2009, 74, 7411-7416.

(28) (a) Vessieres, A.; Jaouen, G.; Gruselle, M.; Rossignol, J. L.; Savignac, M.; Top, S.; Greenfield, S. J. Steroid Biochem. 1988, 30, 301-305; (b) Lang, H.; Köhler, K.; Emmerich, C. Z. Naturforsch, B., J. Chem. Sci. 1995, 50, 923-930.

(29) Kuhn, O.; Rau, D.; Mayr, H. J. Am. Chem. Soc. 1998, 120, 900-907.

(30) Connor, Richard E.; Nicholas, Kenneth M. J. Am. Chem. Soc. 1987, 109, 5749-5759.

(31) Bell, K. H.; McCaffery, L. F. Aust. J. Chem. 1982, 45, 1213-1224. 